\title{
The opening of wind-driven polynyas
}

\author{
Miguel A. Morales Maqueda ${ }^{1, \dagger}$, A. J. Willmott ${ }^{1,3}$ and I. A. Walkington ${ }^{2}$ \\ ${ }^{1}$ National Oceanography Centre, Liverpool L3 5DA, UK \\ ${ }^{2}$ School of Engineering, The University of Liverpool, Liverpool L69 3GH, UK \\ ${ }^{3}$ School of Physical and Geographical Sciences, Keele University, Staffs ST5 5BG, UK
}

(Received 29 July 2013; revised 27 November 2013; accepted 24 February 2014; first published online 31 March 2014)

The opening of wind-driven coastal polynyas has often been investigated using idealised flux models. Polynya flux models postulate that the boundary separating the region of thin ice adjacent to the coast within the polynya from the thicker ice piling up downstream is a mathematical shock. To conserve mass, any divergence of the ice flux across the shock translates into a change in the shock's position or, in other words, a change in the width of the thin-ice region of the polynya. Polynya flux models are physically incomplete in that, while they conserve ice mass, they do not conserve linear momentum. In this paper, we investigate the improvements that can be achieved in the simulation of polynyas by imposing conservation of momentum as well as mass. We start by adopting a mathematically solid formulation of the ice mass and momentum balances throughout the polynya region, from the coast to the pack ice. Hydrostatic and plastic versions of the ice internal forces are used in the model. Two different approaches are then explored. We first postulate the existence of a shock at the seaward edge of the thin-ice region of the polynya and derive jump conditions for the conservation of ice mass and momentum at the shock which are consistent with the continuous model physics. Polynyas simulated by this mass- and momentum-conserving shock model always reach a steady state if the polynya forcing is uniform in space and constant in time. This is also true for all polynya flux models presented previously in the literature, but the location of the steady-state polynya edge and the time required to reach it can greatly differ between shock formulations and more simplistic flux ones. We next relax the assumption that a shock exists and let the boundary between thin ice and piling up ice emerge naturally as part of the full solution of the continuous model equations. Polynyas simulated in this way are very different from those simulated by either shock or flux models. Most notably, we find that steady-state polynya solutions are not always attainable in the continuous model. We determine under which conditions this is so and explain how such unsteady solutions come about. We also show that, in those cases when a steady-state solution exists in the continuous model, the steady-state polynya width is considerably larger than in the shock model, and the time required to attain it is accordingly longer. The occurrence of such significant differences between the polynya solutions calculated with flux and shock models, on the one hand, and with more sophisticated continuous 
formulations, on the other hand, suggests that the former are, at best, incomplete, and should be used with caution.

Key words: ocean circulation, rheology, sea ice

\section{Introduction}

Wind-driven coastal polynyas are regions of open water or new ice adjacent to the shoreline that form in frozen polar oceans when offshore winds propel the sea ice away from the coast. The offshore extension, or width, of a typical polynya ranges from a few hundred metres to a few thousand metres. Polynyas extend alongshore over distances that are usually ten to a hundred times the polynya width. In spite of their relatively small size, polynyas have a considerable climatic importance because of their effects on surface heat, moisture and gas exchanges (Miller \& DiTullio 2007; Minnett \& Key 2007), sea ice growth and dense water formation (Williams, Carmack \& Ingram 2007) and biological activity, from primary production (Tremblay \& Smith 2007) to marine birds and mammals (Stirling 1997; Karnovsky, Ainley \& Lee 2007).

Three regions can be distinguished in a wind-driven coastal polynya: an open-water and thin-ice region, a pile-up region and a consolidating and new-ice region (figure 1). The first region, hereafter referred to as the 'thin-ice region', is adjacent to the coast. Ice crystals with sizes ranging from a few tenths of a millimetre to a few millimetres (Smedrud \& Skogseth 2006; Morse \& Richard 2009) form in the open water as it loses heat to the cold atmosphere. These ice crystals, also called frazil ice, are kept in suspension within the upper few metres of the water column, although they tend to ascend, or precipitate, to the surface as they gain buoyancy forming a thin layer of ice termed grease ice. At or near the surface, frazil ice tends to move in free drift, which, ignoring Stokes drift and Coriolis effects, is the motion resulting from a balance between air-ice and ice-ocean stresses. Frazil ice is thus herded seaward and eventually piles up downstream against pre-existing ice. Thermodynamic and dynamic processes cause the frazil ice thus piled up to consolidate into ice pancakes or to accrete alongside trailing ice floes in the consolidating and new-ice region, hereafter referred to as the 'new-ice region', which extends offshore from the pile-up region to merge into the pack ice. Ice motion in this region tends to be constrained by internal ice stresses that force the ice to slow down until its velocity matches that of the pack ice. It is not uncommon in the literature to see the term 'polynya' used to refer solely to the thin-ice region of a polynya. Although imprecise, this terminology is often adopted as a convenient shorthand, and we will use it sometimes in this paper when there is no ambiguity as to its meaning.

Certain aspects of the dynamics of wind-driven polynyas can be modelled using state-of-the-art, dynamic-thermodynamic sea-ice models. However, these models are computationally expensive, they rarely afford high enough resolutions for an adequate representation of coastal polynyas, and contain only, if at all, crude parameterisations of the processes of frazil ice growth, transport, pile-up and consolidation. A realistic polynya model must be able to describe the following processes. First, the formation of frazil ice crystals of different sizes within the supercooled oceanic mixed layer and their subsequent precipitation toward the sea surface (Svensson \& Omstedt 1998; Holland \& Feltham 2005). Second, the offshore transport of surface and near-surface frazil ice, which has contributions from Stokes drift and associated Langmuir circulations (Pease 1987; Martin 2001), as well as from Ekman and geostrophic flows. Third, the viscous hardening of the frazil ice-water mixture as 

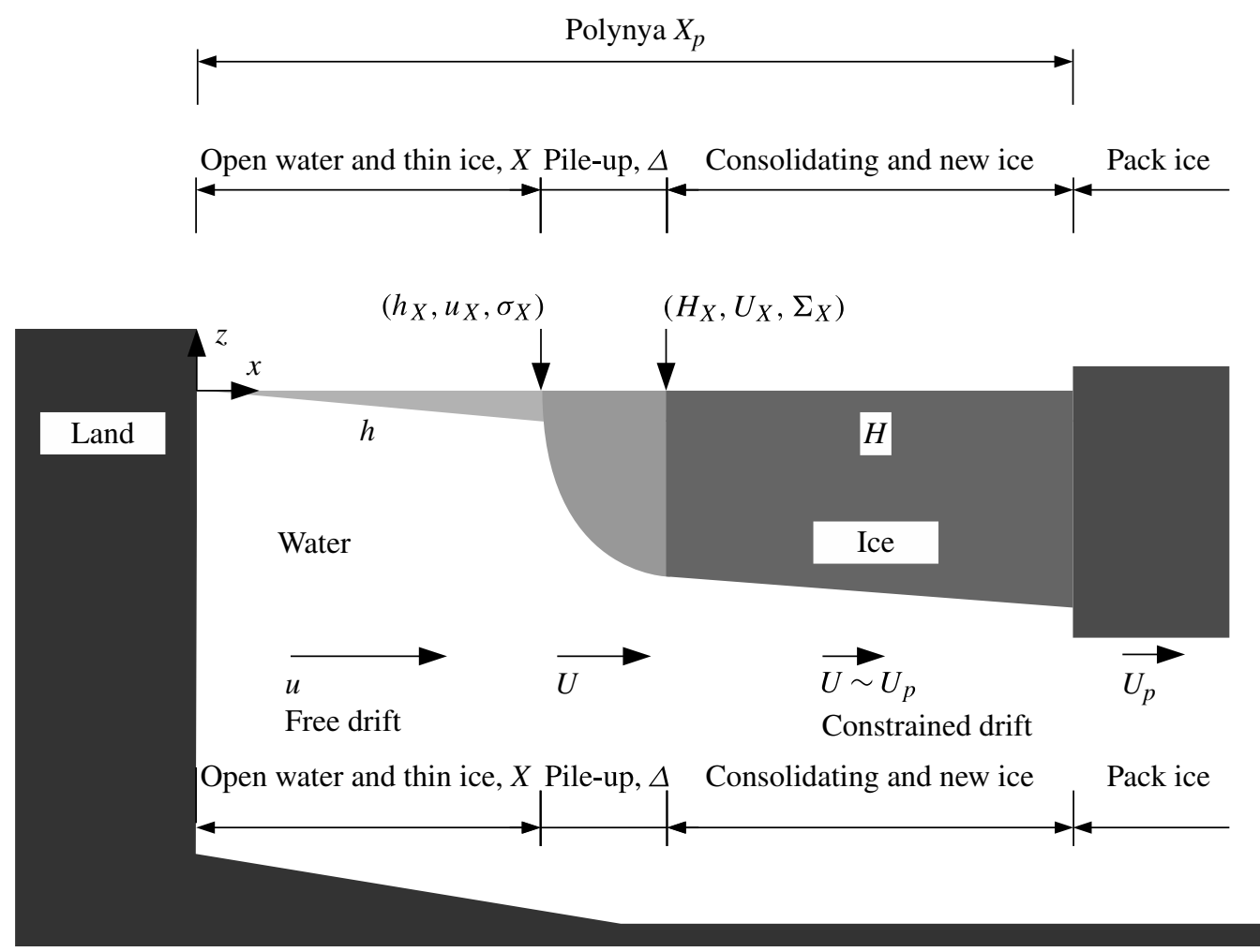

FIGURE 1. Schematic of an opening wind-driven polynya showing the open-water and thin-ice, pile-up and consolidating and new-ice regions. The widths of the thin-ice and pile-up regions are represented by $X$ and $\Delta$, respectively, while $X_{p}$ is the distance from the land to the pack ice, i.e. the total width of the polynya. The thickness of frazil ice in the thin-ice region is denoted by $h$, and the thickness of piling up and new ice is denoted by $H$. The corresponding velocities are denoted by $u$ (typically, close to free drift) and $U$ (normally, appreciably smaller than $u$ because of internal ice constraints), respectively. The pack-ice velocity is denoted by $U_{p}$. The ice thickness, velocity and vertically integrated internal stress on the upstream (downstream) end of the pile-up region, $h_{X}, u_{X}$ and $\sigma_{X}\left(H_{X}, U_{X}\right.$ and $\left.\Sigma_{X}\right)$, respectively, are shown too. The ice velocity in the new-ice region is expected to be close in value to the pack-ice velocity, as indicated.

the volumetric ice concentration increases. Modelling of this hardening process and its impact on surface gravity wave propagation and attenuation requires the formulation of a suitable ice rheology at low ice concentrations, for which there is no definitive consensus (Martin \& Kauffman 1981; Newyear \& Martin 1997; Keller 1998; De Carolis, Olla \& Pignagnoli 2005; Smedrud 2011). Fourth, the piling up and consolidation of frazil ice against existing ice pancakes and ice floes along the seaward boundary of the thin-ice region, a process which depends on the physics of the wavefield, particularly wave radiation stress dynamics (Martin \& Kauffman 1981), and on the rheological (Martin \& Kauffman 1981; Bauer \& Martin 1983; Smedrud 2011) and thermodynamic (Doble, Coon \& Wadhams 2003) characteristics of the ice on either side of the pile-up region. The incorporation of all these processes in a comprehensive polynya model is a daunting task that remains yet to be attempted. 
Polynya flux models endeavour to fill the gap between dynamic-thermodynamic sea-ice models, which, while widely used nowadays, remain very incomplete from the point of view of the representation of wind-driven polynyas, and all-inclusive polynya models, which, however desirable, do not yet exist. Flux models exploit simplifying assumptions on how frazil ice transport, pile-up and consolidation occur in a polynya (e.g. Pease 1987; Ou 1988; Willmott, Holland \& Morales Maqueda 2007). Although these models can, in all generality, be formulated in two spatial dimensions, the essential principles of the flux model approach can be fully captured in a one-dimensional context, which is as follows. Assume that the polynya is initially closed and that, for simplicity, the pack ice is moving with known constant velocity $U_{p}$ in the $x$ direction (figure 1). The location of the boundary between the polynya and the pack ice is therefore always known and is given by $X_{p}=U_{p} t$, where $t$ is the time elapsed from the moment when the pack ice started to drift offshore. As the polynya opens, all three polynya regions, namely the thin-ice, pile-up and new-ice regions, widen, but the pile-up region remains relatively narrow in comparison to the other two regions. If the width of the pile-up region can be neglected, then an expression for the rate of change of the width of the thin-ice region, $X$, can be derived from the principle of mass conservation, namely,

$$
h_{X}\left(u_{X}-\frac{\mathrm{d} X}{\mathrm{~d} t}\right)=H_{X}\left(U_{X}-\frac{\mathrm{d} X}{\mathrm{~d} t}\right),
$$

where $h_{X}$ and $u_{X}$ are the thickness and velocity, respectively, of frazil ice arriving at the pile-up region, and $H_{X}$ and $U_{X}$ are the thickness and velocity, respectively, of ice exiting the pile-up region (Ou 1988). Frazil ice and water are mixed in the upper ocean at volumetric concentrations that vary in space and time and, even when the frazil ice has fully precipitated to the surface, its concentration is neither uniform nor constant. Therefore, $h_{X}$ and $H_{X}$ in (1.1) do not represent actual ice thicknesses but rather effective ones. We define them as $h_{X}=m_{X} / \rho_{i}$ and $H_{X}=M_{X} / \rho_{i}$, where $m_{X}$ and $M_{X}$ are the mass of ice per unit area to the left and right of the pile-up region, respectively, and $\rho_{i}$ is a reference ice density $\left(950 \mathrm{~kg} \mathrm{~m}^{-3}\right.$, say). To predict the evolution of $X$ with the help of (1.1), $h_{X}, u_{X}, H_{X}$ and $U_{X}$ need to be somehow calculated or prescribed. Typically, $u_{X}$ is obtained from simple formulations of the ice linear momentum balance, such as, for example, the free-drift approximation, which postulates a balance between wind-ice and ice-ocean stresses. If the frazil ice velocity and growth rate in the thin-ice region of the polynya are uniform and steady, then $h_{X}=F t_{X}$, where $F$ is the frazil ice growth rate, $t_{X}=X / u$ is the time it takes for frazil ice to cross the thin-ice region, and $u=u_{X}$ is the frazil ice velocity. The momentum balance in the new-ice region is assumed to be such that the ice is constrained to move with a velocity close or equal to that of the pack ice, and so $U_{X} \approx U_{p}$. Finally, $H_{X}$ is typically either prescribed (Pease 1987; Ou 1988), parameterised as a function of various oceanic and atmospheric variables such as polynya fetch and wind speed (Alam \& Curry 1998; Winsor \& Björk 2000), or derived from simple hydrodynamic models of the frazil ice-water mixture (Bauer \& Martin 1983; Biggs, Morales Maqueda \& Willmott 2000).

Irrespective of how the pile-up thickness, $H_{X}$, is calculated, all flux models proposed to date are such that, under steady wind forcing and frazil ice growth rates, the thinice region of the polynya eventually reaches a steady-state width, $\underline{X}$. When the width $\underline{X}$ is attained, ice production within the thin-ice region of the polynya is balanced by ice export across $x=\underline{X}$, namely, $\int_{0}^{\underline{X}} F \mathrm{~d} x=H_{X} U_{X}$, an idea first suggested by Lebedev (1968). 
Although flux models have proven useful in both theoretical and applied polynya studies (Morales Maqueda, Willmott \& Biggs 2004; Willmott et al. 2007), the reduced physics invoked by these models lends itself to many criticisms. Prominent among these is the fact that, while flux models conserve mass across the pile-up region, as guaranteed by (1.1), they ignore the conservation of linear momentum, an approach which simplifies the mathematics of the model but can be justified on no grounds other than expediency. To correct this deficiency, a momentum conservation condition of the form

$$
h_{X} u_{X}\left(u_{X}-\frac{\mathrm{d} X}{\mathrm{~d} t}\right)-\frac{\sigma_{X}}{\rho_{i}}=H_{X} U_{X}\left(U_{X}-\frac{\mathrm{d} X}{\mathrm{~d} t}\right)-\frac{\Sigma_{X}}{\rho_{i}},
$$

is introduced in this paper to supplement (1.1). In (1.2), $\sigma_{X}$ and $\Sigma_{X}$ are the vertically integrated ice internal stresses immediately upstream and downstream of the pile-up region, respectively. As the alert reader will have noticed, equations (1.1) and (1.2) are the Rankine-Hugoniot shock, or jump, conditions for a one-dimensional system with vertically integrated internal stress $\sigma$ (e.g. Godlewski \& Raviart 1996). The polynya formulation based on (1.1) and (1.2) can therefore be aptly called a "polynya shock model'. Equations (1.1) and (1.2) permit the calculation of $H_{X}$ and $\mathrm{d} X / \mathrm{d} t$ as functions of $u_{X}, h_{X}$ and $U_{X}$ in a dynamically consistent way. A more detailed discussion of this polynya shock model will be given in $\S 3$.

While dynamically sounder than polynya flux models, the shock model introduced above shares with the former models a serious shortcoming, namely, that both prescribe, rather than predict, $U_{X}$. Typically, the choice $U_{X}=U_{p}$ is made. The rationale for this choice lies on the notion that ice in the new-ice region quickly becomes hardened enough to move in unison with the pack ice. However, it is not clear why this must be so in general. A balance of forces such that different parts of the new-ice region would move with different velocities is perfectly conceivable. Therefore, the ice momentum budget in this region of the polynya should be predicted, not prescribed. The conditions determining whether the new-ice region moves as a rigid body or as a yielding continuum are investigated in this paper based on a simple, continuous formulation of the ice mass and momentum balance equations of the entire polynya. We also discuss the existence and properties of steady-state and unsteady polynya solutions derived from such a model.

This paper is organised as follows. Section 2 describes the polynya continuous model formulation. Section 3 shows how the polynya shock model with jump equations (1.1) and (1.2) can be derived from the continuous model after invoking a number of simplifying assumptions. This section discusses as well the sensitivity of the polynya shock solutions to varying frazil ice production rates and ice velocities. Length and time scales for the opening of a polynya are also derived from this model. Section 4 returns to the continuous model and similarly explores the model sensitivity to variations in the ice growth rate, wind stress and pack-ice velocity. Different steadystate and unsteady polynya-opening regimes that depend on both polynya forcing and ice rheology parameters are identified. Continuous and shock model solutions are also compared in this section. In $\S 5$ we put forward five important questions pertaining to the continuous model and provide prospective answers. These questions are as follows. (i) Is it possible to characterise in a simple way the various polynya-opening regimes of the continuous model in terms of polynya forcing parameters, boundary conditions and ice properties? (ii) Are these different regimes all realisable in practice in realistic polynyas? (iii) What is the leading momentum balance in each of the three 
regions of a polynya? (iv) Can these different momentum balances be used to develop polynya models of a reduced complexity compared with that of the full continuous formulation? (v) How does the choice of ice rheology affect the polynya solutions? Section 6 concludes the paper with a summary and final remarks.

\section{Continuous model formulation}

The dynamics of wind-driven coastal polynyas are too complex and too poorly known to be easily reduced to mathematical expressions. We introduce here a one-dimensional model that is sophisticated enough to allow us to simulate directly the formation and evolution in time of all three regions of a polynya. The model is sufficiently simple to remain mathematically tractable, but of the right level of complexity to capture key mechanisms in the opening of a wind-driven polynya.

The model (figure 1) comprises a coastline situated at $x=0$, a pre-existing pack ice that is adjacent to the coast at $t=0$ and moves away from the coast with constant velocity $U_{p}$ (here $U_{p}$ is prescribed as a boundary condition but, in reality, it results from the balance of momentum in the pack-ice region, which could, in principle, be calculated), and an oceanic active layer consisting of a mixture of frazil ice and seawater that extends from the coast to the pack-ice boundary and floats over a motionless and infinitely deep ocean. The mass and momentum conservation equations for ice in the mixture are

$$
\begin{gathered}
\frac{\partial h}{\partial t}+\frac{\partial(h u)}{\partial x}=F, \\
\frac{\partial(h u)}{\partial t}+\frac{\partial(h u u)}{\partial x}=\frac{1}{\rho_{i}}\left(\frac{\partial \sigma}{\partial x}+\alpha\left(\tau_{s}+\tau_{b}\right)+\tau_{i n t}+\frac{\partial}{\partial x}\left(h M \frac{\partial u}{\partial x}\right)\right),
\end{gathered}
$$

where $h$ is the effective ice thickness (i.e. the thickness of ice that would result from the consolidation of all of the frazil ice contained in the active layer at a density $\rho_{i}$ ), $u$ is the active layer velocity (both frazil ice and water in the active layer are assumed to move with the same velocity), $F$ is the ice production rate, $\sigma$ is the vertically integrated internal stress of the layer (see below), $\alpha$ is the volumetric concentration of frazil ice in the layer, $\tau_{s}$ is wind stress and $\tau_{b}$ is the stress at the base of the layer. Truer to reality, the surface and bottom stresses on the ice should be described by $\alpha_{s} \tau_{s}$ and $\alpha_{b} \tau_{b}$, respectively, where $\alpha_{s}$ is the fraction of the surface traction experienced by the ice component, and similarly for $\alpha_{b}$ at the bottom. For simplicity, we assume here that $\alpha=\alpha_{s}=\alpha_{b}$. Typically, $\alpha$ will be small near the coast and will approach one in the pile-up and new-ice regions. However, for the sake of keeping the model formulation as simple as possible, we adopt a constant value for $\alpha$ in this study, which means that, as ice grows, the active layer must entrain water at a volumetric rate of $F / \alpha$ in order to ensure that $\alpha$ does not change during the evolution of the system. In practice, the numerical simulations presented below use $\alpha=1$, which is equivalent to assuming that all ice precipitates to the surface or to the base of a pre-existing ice layer immediately upon its formation, the model thus becoming a 1(1/2)-layer model where the active layer is ice. The term $\tau_{\text {int }}$ represents the drag associated with the fact that ice and water have in general a non-zero relative velocity within the active layer. Quite a number of parameterisations of $\tau_{\text {int }}$ have been proposed (e.g. Manninen, Taivassalo \& Kallio 1996), but we will not concern ourselves here with them since, under our stated assumption that $\alpha=1$, the water-ice interaction force becomes zero. As $h$ increases and the ocean becomes more insulated from the atmosphere, the ice 
production rate $F$ is expected to steadily decay. A simple formulation for $F$, inspired by Morales Maqueda et al. (1998), is

$$
F=F_{0} \frac{\kappa}{\kappa+v h},
$$

where $F_{0}$ is the ice production rate over ice-free waters, $\kappa$ is the thermal conductivity of ice and $v$ is a Haney-type air-ice exchange coefficient (Haney 1971). The horizontal viscosity term on the right-hand side of $(2.1 b)$ could be construed as representing the impact of horizontal eddies and other small-scale flows (e.g. Langmuir circulations) on the momentum balance. According to numerical experiments with three-dimensional ocean models, baroclinic eddies control the export of dense water formed in a polynya (e.g. Wilchinsky \& Feltham 2008), and we do not discard that they might play a role in the dynamics of polynyas, especially mature ones, should they open to sizes larger than the local baroclinic Rossby radius. However, we assume here that the ocean is in a state in which horizontal eddy mixing and viscosity are small, and take $M$ such that, while ensuring that upstream and downstream boundary conditions for $u$ can be imposed at all times (see (2.8) below), eddy effects do not play a significant role in the dynamics of the active layer outside narrow viscous boundary layers at $x=0$ and $x=U_{p} t$. In $(2.1 b), \tau_{s}$ is prescribed, while the bottom stress is given by

$$
\tau_{b}=-\rho_{w} c_{D}|u| u,
$$

where $\rho_{w}$ is the density of sea water and $c_{D}$ is a drag coefficient. The vertically integrated stress tensor, $\sigma$, has been represented in some previous studies as a hydrostatic pressure term (e.g. Bauer \& Martin 1983; Biggs et al. 2000), namely,

$$
\sigma=-P^{\star} h^{2},
$$

where $P^{\star}=\rho_{m} g^{\prime} / 2$, in which

$$
\rho_{m}=\alpha \rho_{i}+(1-\alpha) \rho_{w}
$$

is the average density of the active layer, and the reduced gravity is given by

$$
g^{\prime}=g \frac{\rho_{w}-\rho_{m}}{\rho_{w}},
$$

where $g$ is the acceleration of gravity. The formulation (2.4) is appropriate only in cases when the concentration of frazil ice in the active layer is low, but becomes increasingly inadequate as $\alpha$ grows, and so it is unsuitable for modelling the polynya dynamics in the pile-up and new-ice regions. For large $\alpha$, a viable approach is to treat the active layer, which, if $\alpha \approx 1$, will consist mostly of consolidated ice, as a plastic medium with vertically integrated internal stresses given by

$$
\left.\begin{array}{l}
\sigma=0 \quad \text { if } \frac{\partial u}{\partial x}>0, \\
-P^{\star} h^{n} \leqslant \sigma \leqslant 0 \quad \text { if } \frac{\partial u}{\partial x}=0, \\
\sigma=-P^{\star} h^{n} \quad \text { if } \frac{\partial u}{\partial x}<0,
\end{array}\right\}
$$


where $P^{\star}$ and $n \geqslant 1$ are constants. Typical values for $n$ used in the literature are $n=1$ (Hibler 1979), $n=3 / 2$ (Hopkins 1998; Wilchinsky, Feltham \& Miller 2006) and $n=2$ (Rothrock 1975; Overland \& Pease 1988). Note that some of the parameterisations just cited use a $P^{\star}$ that is not constant but depends on other ice field variables. In a non-divergent situation, the ice will offer as much resistance as is needed to prevent convergence, which may require weaker stresses than the maximum possible one, hence the ambiguity in the value of $\sigma$ in this case. The boundary conditions are

$$
\left.\begin{array}{ll}
u=0, & \text { at } x=0 \\
u=U_{p}, & \text { at } x=U_{p} t .
\end{array}\right\}
$$

The boundary condition at $x=0$ automatically guarantees that there is no flux of mass across the land boundary. When the ice is diverging from the coast, its velocity increases rapidly through a very narrow layer spanning only a few grid points from its value of zero at the coast to a nearly uniform value in the thin-ice region. The model described here is primarily intended for the exploration of processes, hence, its simplified physics. Some of the approximations and assumptions that we have adopted reduce considerably the domain of applicability of the model, and so should be revised at some later point. A discussion of six key model simplifications follows.

(i) The Coriolis force is ignored in $(2.1 b)$, a choice that is appropriate as long as the active layer remains thin, but is less so if ice piles up to large depths downstream of the thin-ice region.

(ii) The ice production rate term (2.2) is probably adequate, to first order, for a slab of thin ice, but it does not capture the complexities of the thermodynamic growth of suspended frazil ice (e.g. Holland \& Feltham 2005) or the consolidation of slush and ice rubble in the pile-up and new-ice regions (Marchenko 2008).

(iii) The quadratic bottom stress (2.3), while apt for representing friction underneath consolidated ice, is not quite as suitable for a layer containing suspended frazil ice at low concentrations. For such a layer, a more apposite formulation would be $\tau_{b}=-\rho_{m} m u / h$, where $m$ is a Fickian viscosity.

(iv) The relative velocity between frazil ice particles and fluid within the active layer is assumed to be negligible so that an additional ice-water drag force term internal to the active layer can be set to zero in $(2.1 b)$ (e.g. Manninen et al. 1996).

(v) The treatment of ice internal forces is also highly schematic. A complete polynya model should incorporate a formulation of the transition from the hydrostatic pressure case (2.4), which is appropriate for the description of the active layer dynamics in the thin-ice region of the polynya, to the plastic case (2.7), which will obtain in the pile-up and new-ice regions as the fractional ice content of the ice-water mixture approaches one. However, a theory for the coagulation of frazil ice crystals and their consolidation into solid ice bodies remains yet to be developed. It is tempting to anticipate that viscous, elastic or viscoelastic rheologies with bulk viscosities increasing as the ice volumetric concentration grows, and possibly including a multinomial dependence on $h$ to account for different ice failure pathways (e.g. Wilchinsky \& Feltham 2008), could successfully describe the properties of the ice-liquid system as it hardens. However, it seems prudent to leave the use of sophisticated multirheology ice formulations out of the scope of the paper. Instead, we study the polynya opening process in the hydrostatic and plastic rheology limits separately, in anticipation 
that results from both idealised cases will shed valuable light into the polynya dynamics, but bearing in mind nevertheless that a realistic polynya rheology will be significantly more complicated than either of the idealised rheology cases studied by us.

(vi) Intimately related with the rheology simplifications just mentioned, is the fact that the model assumes that all ice properties, not just rheology, are uniform throughout the polynya. In reality, the thin-ice, pile-up, new-ice and pack-ice regions consist of ice of very different types, whereas, in the model, ice is represented as a material layer whose physical properties are the same in all regions. This approach becomes an important limitation in some cases. For example, the thin-ice region in the model is distinguished from the pile-up and new-ice regions solely by the fact that it is adjacent to the coast and its momentum balance is predominantly free drift. However, the ability to move in free drift is not exclusively reserved to thin ice or to ice at very low areal concentrations. A thick, continuous ice cover can also move in near free drift under appropriate circumstances. And we shall see below that ice in what we call the 'thin-ice region' can indeed in some cases grow arbitrarily thick while the internal stress gradients remain small. When this happens, the use of the term 'thin-ice region' is obviously misleading. All of these simplifications are adopted here to facilitate focusing on key model features. At a later stage, more realistic polynya formulations will have to be used.

Solutions of (2.1)-(2.4), with $\sigma$ given by either (2.4) or (2.7), have to be found numerically, but, before this is attempted, it is practical to cast this system of equations in a non-dimensional form. To do so, let us define a horizontal length scale, $x_{c}$, a time scale, $t_{c}$, an effective ice thickness scale, $h_{c}$ and an ice velocity scale, $u_{c}$, with associated non-dimensional variables given by

$$
\tilde{x}=\frac{x}{x_{c}}, \quad \tilde{t}=\frac{t}{t_{c}}, \quad \tilde{h}=\frac{h}{h_{c}}, \quad \tilde{u}=\frac{u}{u_{c}} .
$$

We choose the following scales:

$$
h_{c}=2 \frac{\kappa}{v}, \quad u_{c}=\left(\frac{P^{\star}}{\rho_{i}} h_{c}^{n-1}\right)^{1 / 2}, \quad t_{c}=\frac{h_{c}}{F_{0}}, \quad x_{c}=u_{c} t_{c} .
$$

In (2.10), the scale $h_{c}$ is the thickness at which ice heat loss to the atmosphere is twice the heat conduction through ice for identical ice-ocean and air-ice temperature contrasts, i.e. $2 \kappa \delta T / h_{c}=v \delta T$. The introduction of the arbitrary factor 2 in the definition of $h_{c}$ leads to simplifications in some of the expressions discussed later in $\S 4$. The scale $u_{c}=c / \sqrt{n}$, where $c$ is the phase speed of surface gravity waves, or linear compression waves, depending on the nature of the internal forces, for an ice thickness $h_{c}$. The interpretations of the scales $t_{c}$ and $x_{c}$ are obvious.

The use of these four scales leads to the non-dimensional equations

$$
\begin{aligned}
\frac{\partial \tilde{h}}{\partial \tilde{t}}+\frac{\partial(\tilde{h} \tilde{u})}{\partial \tilde{x}} & =\frac{1}{1+2 \tilde{h}}, \\
\frac{\partial(\tilde{h} \tilde{u})}{\partial \tilde{t}}+\frac{\partial(\tilde{h} \tilde{u} \tilde{u})}{\partial \tilde{x}} & =\frac{\partial \tilde{\sigma}}{\partial \tilde{x}}+A\left(\tilde{\tau}_{s}-|\tilde{u}| \tilde{u}\right)+\tilde{\tau}_{i n t}+\frac{\partial}{\partial \tilde{x}}\left(\tilde{h} \tilde{M} \frac{\partial \tilde{u}}{\partial \tilde{x}}\right),
\end{aligned}
$$

where $\tilde{\sigma}$ obeys the same equation as the dimensional stress tensor, $\sigma$ (i.e. (2.4) or (2.7) depending on the case studied), but with $P^{\star}$ replaced by one, and $h, u$ and $x$ replaced by their corresponding non-dimensional variables. In $(2.11 b)$, the 
non-dimensional coefficient $A=\alpha\left(\rho_{w} / \rho_{i}\right) c_{D}\left(u_{c} / F_{0}\right)$, the non-dimensional wind stress $\tilde{\tau}_{s}=\tau_{s} /\left(\rho_{w} c_{D} u_{c}^{2}\right)$, the non-dimensional interaction drag $\tilde{\tau}_{\text {int }}=t_{c} /\left(\rho_{i} h_{c} u_{c}\right) \tau_{\text {int }}$ and the non-dimensional viscosity $\tilde{M}=M / \rho_{i} t_{c} / x_{c}^{2}$.

In $\S 4$, we will discuss the properties of polynya solutions obtained by solving (2.1)-(2.4) with $\sigma$ given by either (2.4) or (2.7), but it will prove instructive to first discuss the conceptual connection between this system of equations and the shock model equations (1.1) and (1.2) discussed in the introduction.

\section{Polynya shock model}

If the solutions of the continuous model described above were qualitatively similar to the idealised polynya solution depicted in figure 1 , it would seem natural to try to approximate the ice pile-up as a mathematical jump in ice thickness and velocity. Assuming that $\tilde{M}$ in $(2.11 b)$ is sufficiently small that horizontal viscosity at the jump can be neglected, the integration of (2.11a) and (2.11b) over the infinitesimally small region $\tilde{X} \leqslant \tilde{x} \leqslant \tilde{X}+\tilde{\Delta}$, where $\tilde{X}$ and $\tilde{\Delta}$ are the non-dimensional widths of the thin-ice and pile-up regions, respectively, leads to the jump conditions:

$$
\begin{gathered}
\tilde{h}_{\tilde{X}}\left(\tilde{u}_{\tilde{X}}-\frac{\mathrm{d} \tilde{X}}{\mathrm{~d} \tilde{t}}\right)=\tilde{H}_{\tilde{X}}\left(\tilde{U}_{\tilde{X}}-\frac{\mathrm{d} \tilde{X}}{\mathrm{~d} \tilde{t}}\right), \\
\tilde{h}_{\tilde{X}} \tilde{u}_{\tilde{X}}\left(\tilde{u}_{\tilde{X}}-\frac{\mathrm{d} \tilde{X}}{\mathrm{~d} \tilde{t}}\right)-\tilde{\sigma}_{\tilde{X}}=\tilde{H}_{\tilde{X}} \tilde{U}_{\tilde{X}}\left(\tilde{U}_{\tilde{X}}-\frac{\mathrm{d} \tilde{X}}{\mathrm{~d} \tilde{t}}\right)-\tilde{\Sigma}_{X},
\end{gathered}
$$

which are the non-dimensional equivalents of (1.1)-(1.2) and formally equivalent to the well-known discontinuity, jump or shock conditions of the shallow-water equations in hydrodynamics (e.g. Stoker 1957). We recall that, in (3.1), a variable with the subscript $\tilde{X}$ is evaluated at $\tilde{x}=\tilde{X}$ and that lowercase and uppercase symbols refer to variable values upstream and downstream of the jump, respectively. As pointed out in the introduction, equation (3.1) can be used to calculate the width of the thin-ice region of the polynya, $\tilde{X}$, and the thickness of ice after pile-up, $\tilde{H}_{\tilde{X}}$, if suitable assumptions are made regarding $\tilde{h}_{\tilde{X}}, \tilde{u}_{\tilde{X}}$ and $\tilde{U}_{\tilde{X}}$. If we assume that, in the thin-ice region, frazil ice is approximately moving in free drift (i.e. surface and bottom stresses are in balance, namely, $\tilde{\tau}_{s}-|\tilde{u}| \tilde{u}=0$ ) and ice production is approximately uniform and constant $(\tilde{F} \approx 1)$, then

$$
\begin{aligned}
& \tilde{u}_{\tilde{X}} \approx \frac{\tilde{\tau}_{s}}{\sqrt{\left|\tilde{\tau}_{s}\right|}}, \\
& \tilde{h}_{\tilde{X}} \approx \frac{\tilde{X}}{\tilde{u}_{\tilde{X}}} .
\end{aligned}
$$

In addition, since there is flow convergence at all times at $\tilde{x}=\tilde{X}$, the internal stresses at $\tilde{x}=\tilde{X}$ are

$$
\begin{gathered}
\tilde{\sigma}_{\tilde{X}}=-\tilde{h}_{\tilde{X}}^{n}, \\
\tilde{\Sigma}_{\tilde{X}}=-\tilde{H}_{\tilde{X}}^{n} .
\end{gathered}
$$

Finally, we impose

$$
\tilde{U}_{\tilde{X}}=\tilde{U}_{p} \leqslant \tilde{u}_{\tilde{X}},
$$


where $\tilde{U}_{p}$ is the non-dimensional velocity of the pack ice. Inequality (3.4) ensures that there is flow convergence at some point between $\tilde{x}=0$ and $\tilde{x}=\tilde{U}_{p} \tilde{t}$, and that ice pile-up can therefore occur somewhere within the polynya. The nonlinear set of equations (3.1)-(3.4) needs to be integrated numerically, but insight into key properties of the solution can be attained analytically.

Let us first note that $(3.1 a)$ requires that the quantities $\tilde{u}_{\tilde{X}}-\mathrm{d} \tilde{X} / \mathrm{d} \tilde{t}$ and $\tilde{U}_{\tilde{X}}-\mathrm{d} \tilde{X} / \mathrm{d} \tilde{t}$ be of the same sign. They must, in fact, both be positive since, if they were negative, we would have $\tilde{U}_{\tilde{X}} \leqslant \tilde{u}_{\tilde{X}} \leqslant \mathrm{~d} \tilde{X} / \mathrm{d} \tilde{t}$, and so the ice formed in the thin-ice region of the polynya could never reach the jump, which, therefore, could not have formed in the first place. Consequently, it must be the case that $\mathrm{d} \tilde{X} / \mathrm{d} \tilde{t} \leqslant \tilde{U}_{\tilde{X}} \leqslant \tilde{u}_{\tilde{X}}$.

We now derive two important flow constraints. First, using $(3.1 a)$ to eliminate $\tilde{H}_{\tilde{X}}$ from $(3.1 b)$ and denoting $r=\left(\tilde{u}_{\tilde{X}}-\mathrm{d} \tilde{X} / \mathrm{d} \tilde{t}\right) /\left(\tilde{U}_{\tilde{X}}-\mathrm{d} \tilde{X} / \mathrm{d} \tilde{t}\right) \geqslant 1$ leads, after some manipulations, to

$$
\left(\tilde{u}_{\tilde{X}}-\frac{\mathrm{d} \tilde{X}}{\mathrm{~d} \tilde{t}}\right)\left(\tilde{U}_{\tilde{X}}-\frac{\mathrm{d} \tilde{X}}{\mathrm{~d} \tilde{t}}\right)=\frac{r^{n}-1}{n(r-1)} \tilde{c}_{\tilde{X}}^{2} \geqslant \tilde{c}_{\tilde{X}}^{2},
$$

where

$$
\tilde{c}_{\tilde{X}}=\sqrt{n \tilde{h}_{\tilde{X}}^{n-1}} .
$$

The quantity $\tilde{c}_{\tilde{X}}$ is the non-dimensional phase speed of unforced linear waves immediately upstream of the jump. In the hydrostatic case, these waves are internal gravity waves. In the plastic rheology case, they are compression waves. We can also eliminate $\tilde{h}_{\tilde{X}}$ from (3.1b), using again (3.1a) and writing $s=1 / r=$ $\left(\tilde{U}_{\tilde{X}}-\mathrm{d} \tilde{X} / \mathrm{d} \tilde{t}\right) /\left(\tilde{u}_{\tilde{X}}-\mathrm{d} \tilde{X} / \mathrm{d} \tilde{t}\right) \leqslant 1$, to obtain

$$
\left(\tilde{u}_{\tilde{X}}-\frac{\mathrm{d} \tilde{X}}{\mathrm{~d} \tilde{t}}\right)\left(\tilde{U}_{\tilde{X}}-\frac{\mathrm{d} \tilde{X}}{\mathrm{~d} \tilde{t}}\right)=\frac{s^{n}-1}{n(s-1)} \tilde{C}_{\tilde{X}}^{2} \leqslant \tilde{C}_{\tilde{X}}^{2},
$$

where

$$
\tilde{C}_{\tilde{X}}=\sqrt{n \tilde{H}_{\tilde{X}}^{n-1}}
$$

is the non-dimensional phase speed of unforced linear waves immediately downstream of $\tilde{x}=\tilde{X}$. Equations (3.5) and (3.7) make it apparent that, at all times, the jump is a boundary between supercritical upstream flow and subcritical downstream flow. This is a general characteristic of normal shocks (e.g. Massey 1983). According to this shock model, an ice pile-up region, or jump, can occur only if the speed of ice in the thin-ice region of the polynya is larger than the phase speed of the system's unforced linear waves. This is in principle always possible save in the singular case $n=1$, for which the wave phase speed is independent of ice thickness $\left(\tilde{c}_{\tilde{X}}=\tilde{C}_{\tilde{X}}=1\right)$, and (3.5) and (3.7) become identical. It is easy to show that, in this case, polynya solutions with $\mathrm{d} \tilde{X} / \mathrm{d} \tilde{t} \leqslant \tilde{U}_{\tilde{X}} \leqslant \tilde{u}_{\tilde{X}}$ exist only if $1 \leqslant \tilde{u}_{\tilde{X}} \tilde{U}_{\tilde{X}}$, and that these solutions are such that the jump moves offshore with constant speed

$$
\frac{\mathrm{d} \tilde{X}}{\mathrm{~d} \tilde{t}}=\frac{\tilde{u}_{\tilde{X}}+\tilde{U}_{\tilde{X}}-\sqrt{\left(\tilde{u}_{\tilde{X}}+\tilde{U}_{\tilde{X}}\right)^{2}-4\left(\tilde{u}_{\tilde{X}} \tilde{U}_{\tilde{X}}-1\right)}}{2} .
$$

Therefore, no steady-state polynya shock model solution exists when $n=1$. 
Are steady-state solutions possible for $n>1$ ? To answer this question, let us first calculate the polynya solution when $\mathrm{d} \tilde{X} / \mathrm{d} \tilde{t}=0$. At the stationary jump, the effective frazil ice thickness, $\tilde{\tilde{h}}_{\tilde{X}}$, the effective consolidating ice thickness, $\underline{\tilde{H}}_{\tilde{X}}$, and the width of the thin-ice region, $\underline{\tilde{X}}$, are

$$
\begin{aligned}
\underline{\tilde{h}}_{\tilde{X}} & =\left(\frac{\underline{r}-1}{\underline{r}^{n}-1} \tilde{u}_{\tilde{X}} \tilde{U}_{\tilde{X}}\right)^{1 /(n-1)}, \\
\underline{\tilde{H}}_{\tilde{X}} & =\underline{r} \underline{\tilde{h}_{\tilde{X}}}, \\
\underline{\tilde{X}} & =\underline{\tilde{h}}_{\tilde{X}} \tilde{u}_{\tilde{X}},
\end{aligned}
$$

where $\underline{r}=\tilde{u}_{\tilde{X}} / \tilde{U}_{\tilde{X}} \geqslant 1$. Linearising (3.1) around the stationary values in (3.10) leads to

$$
\begin{gathered}
\left(\underline{\tilde{H}}_{\tilde{X}}-\underline{\tilde{h}}_{\tilde{X}}\right) \frac{\mathrm{d} \Delta \tilde{X}}{\mathrm{~d} \tilde{t}}+\tilde{u}_{\tilde{X}} \Delta \tilde{h}_{\tilde{X}}-\tilde{U}_{\tilde{X}} \Delta \tilde{H}_{\tilde{X}}=0, \\
\left(\tilde{u}_{\tilde{X}}^{2}+n \tilde{n}_{\tilde{X}}^{n-1}\right) \Delta \tilde{h}_{\tilde{X}}-\left(\tilde{U}_{\tilde{X}}^{2}+n \underline{\tilde{H}}_{\tilde{X}}^{n-1}\right) \Delta \tilde{H}_{\tilde{X}}=0,
\end{gathered}
$$

where $\Delta \tilde{X}, \Delta \tilde{h}_{\tilde{X}}$ and $\Delta \tilde{H}_{\tilde{X}}$ are small perturbations in $\tilde{X}, \tilde{h}_{\tilde{X}}$ and $\tilde{H}_{\tilde{X}}$, respectively. Eliminating $\Delta \tilde{H}_{\tilde{X}}$ from (3.11a) and (3.11b), and using (3.10c) and the fact that $\Delta \tilde{h}_{\tilde{X}}=1 / \tilde{u}_{\tilde{X}} \Delta \tilde{X}$, the following linearised equation for $\Delta \tilde{X}$ is reached:

$$
\frac{\mathrm{d} \Delta \tilde{X}}{\mathrm{~d} \tilde{t}}=-\frac{(n-1) \underline{r}}{1+\frac{n \underline{r}^{n}(\underline{r}-1)}{\underline{r}^{n}-1}} \frac{\tilde{U}_{\tilde{X}}}{\tilde{\tilde{X}}} \Delta \tilde{X} .
$$

Since the coefficient of $\Delta \tilde{X}$ on the right-hand side of (3.12) is always negative if $n>1$, the stationary solution (3.10) is indeed stable, at least for sufficiently small perturbations, and constitutes therefore a genuine steady state. From (3.12), the nondimensional time scale for the polynya to return to the steady state, $\underline{\tilde{T}}$, is

$$
\underline{\tilde{T}}=\frac{1+\frac{n \underline{r}^{n}(\underline{r}-1)}{\underline{r}^{n}-1}}{(n-1) \underline{r}} \frac{\underline{\tilde{X}}}{\tilde{U}_{\tilde{X}}},
$$

which, within the constraints of the linear approximation, provides also a fairly accurate time scale for the opening of the polynya. For example, if the polynya opening time is defined as the time that it takes for the width of the thin-ice region to reach a fraction $1-\epsilon$ of the steady-state width, $\underline{\underline{X}}$, then the actual opening time obtained by numerically solving (3.1)-(3.4) with either $n=2$ or $n=3 / 2$ and for $\tilde{u}_{\tilde{X}}$ and $\underline{r}$ varying within the ranges $(0,10)$ and $(1,6)$, respectively, is always within approximately $10 \%$ of $-\tilde{T} \ln \epsilon$ for $\epsilon=0.01$, with $\tilde{T}$ always slightly larger than the true opening time (see figure $2 a, c$ ). The ranges of $\tilde{u}_{\tilde{X}}$ and $\underline{r}$ used in the simulations whose results are shown in figure 2 are broad enough to encompass a very large number of realistic polynya opening cases.

It is not our purpose to carry out here a detailed comparison between the polynya shock approach and previous polynya flux models, but it will prove instructive to examine the differences between shock model solutions and those calculated using the theory of Biggs et al. (2000), that is also based on shock concepts, but is restricted 
(a) 6

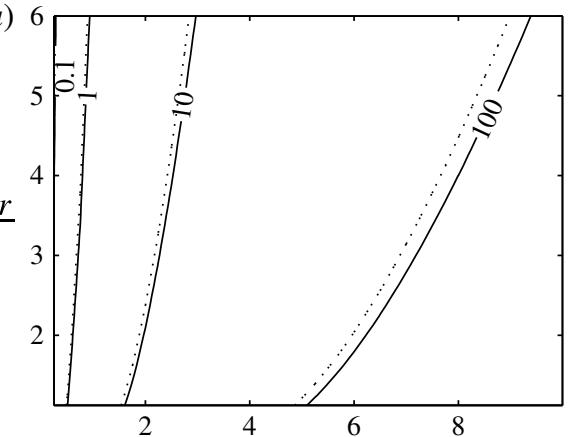

(c)

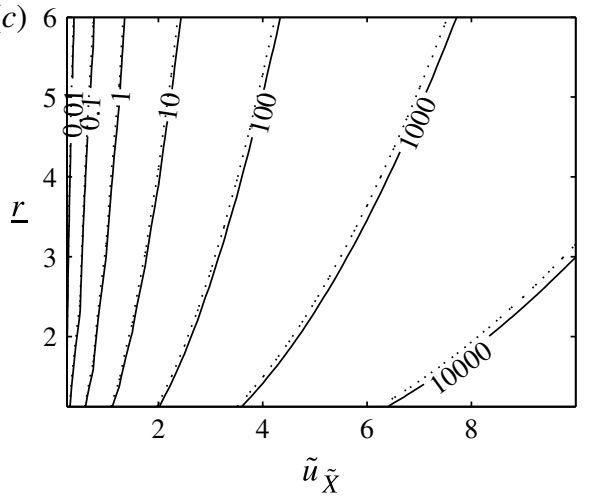

(b)

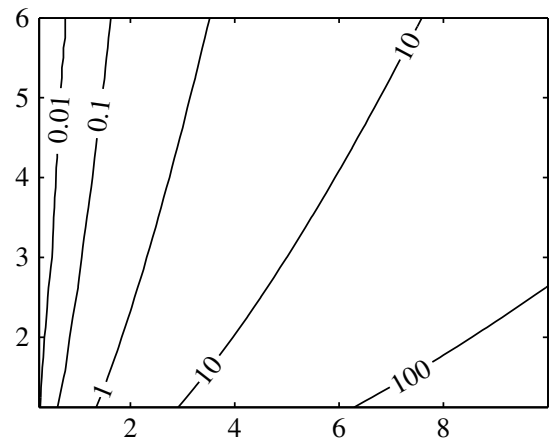

$(d)$

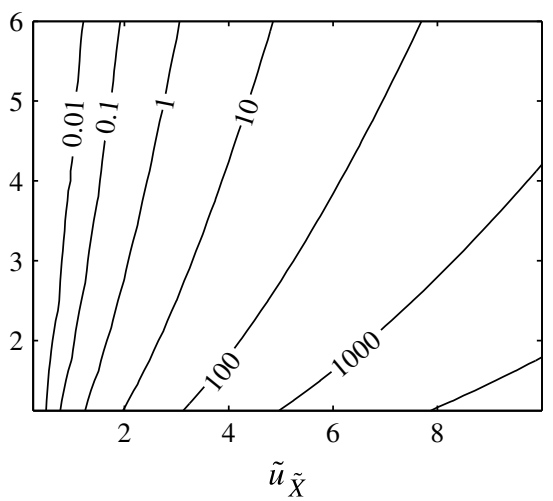

FIgURE 2. Polynya shock model simulations for $n=2$ and $n=3 / 2$. The solutions for $n=2$ apply to both the hydrostatic pressure case and the plastic rheology with quadratic ice strength. Non-dimensional polynya opening time $(a)$ and non-dimensional steady-state polynya width $(b)$ as functions of the non-dimensional frazil ice velocity, $\tilde{u}_{\tilde{X}}$, and the ratio $\underline{r}=\tilde{u}_{\tilde{X}} / \tilde{U}_{\tilde{X}}$ for the case $n=2$. The opening time parameter, $\epsilon$, was 0.01 . The dotted lines in (a) correspond to the opening time estimated using the approximate expression $-\tilde{T} \log \epsilon$, where $\tilde{T}$ is defined in (3.13). Panels $(c)$ and $(d)$ are as $(a)$ and $(b)$, respectively, for the case $n=3 / 2$.

to mass conservation in the hydrostatic case. We will focus on two key parameters, namely, the steady-state polynya width and the opening time.

The dimensional steady-state polynya width in the hydrostatic polynya shock model is obtained from (3.10c) and (2.9)-(2.10). Setting $n=2$ and $P^{\star}=\rho_{m} g^{\prime} / 2$, we obtain the following expression for the steady-state polynya width in the shock model:

$$
\underline{X}=\frac{2 \rho_{i} / \rho_{m}}{F g^{\prime}} \frac{\underline{r}^{2}}{r+1} U_{X}^{3}
$$

The ratio of $\underline{X}$ to the steady-state polynya width of Biggs et al. (2000), which we denote here by $\underline{X}^{B}$ (see their equation (3.6)), is

$$
\frac{\underline{X}}{\underline{X}^{B}} \approx \frac{4 \underline{r}}{\underline{r}^{2}-1}
$$

where we have made the approximation $\rho_{i} / \rho_{m} \approx 1$. The polynya is wider in the shock model than in the flux model of Biggs et al. (2000) if $\underline{r}<2+\sqrt{5} \approx 4.24$. 
Measurements of the relative speeds of frazil and consolidated ice in wind-driven coastal polynyas do not exist, but $u_{X}$ does not probably exceed $U_{X}$ by more than a factor of two or three in most cases, and so we would expect steady-state polynya solutions calculated using the shock method to be generally wider than those derived from the Biggs et al. (2000) flux model. For example, for $\underline{r}=2$, a value frequently used in previous polynya models, $\underline{X} \approx 2.7 \underline{X}^{B}$.

Regarding polynya opening times, a simple calculation shows that, in the hydrostatic case and for $\underline{r} \geqslant 1$, the opening time scale for the polynya shock model is $\underline{T} \approx 2 \underline{X} / U_{X}$. This time scale is to be compared with the corresponding time scale in Biggs et al. (2000), namely, $\underline{T}^{B}=\underline{X}^{B} / U_{X}$, which, in combination with (3.15), leads to

$$
\frac{\underline{T}}{\underline{T}^{B}} \approx \frac{8 \underline{r}}{\underline{r}^{2}-1}
$$

For a typical value $\underline{r}=2$, we have $\underline{T} \approx 5.3 \underline{T}^{B}$, which implies a much slower polynya opening in the shock model.

The shock formulation (3.1)-(3.4) that we have discussed in this section aims at encapsulating the leading physics of the more complex system (2.1)-(2.7). But does it succeed? How well do the polynya shock solutions compare with the solutions of the continuous model?

\section{Continuous model solutions}

In this section, we investigate the solutions of the continuous model (2.11) and compare them with those obtained using the polynya shock model. The system of equations (2.11) is highly nonlinear and has therefore to be solved numerically. Because the boundaries between the different polynya regions are frequently quite narrow (tens to hundreds of metres across), the spatial and temporal resolutions of the model need to be fine, which makes the model computationally expensive. Certain key properties of the solutions to (2.11) can, however, be investigated analytically, which we do in the section below.

\subsection{Existence and properties of steady-state polynya solutions}

We have seen in $\S 3$ that steady-state shock solutions are always possible provided that the ice strength parameter $n$ is greater than one. Is this also the case in the continuous model? We anticipate that a steady-state polynya solution, if it exists, will qualitatively resemble the polynya system schematically depicted in figure 1, with the difference that, in an exact steady state, the pack ice will have drifted away to $x=\infty$ and the region to the right of $x=X$ will be entirely occupied by consolidating new ice. In such a steady state, the widths of the thin-ice and pile-up regions will have reached their time-independent values, and the ice in the new-ice region will be moving offshore with uniform velocity $U=U_{p}$. The non-dimensional, steady-state polynya equations in this region will then reduce to

$$
\begin{aligned}
\tilde{U}_{p} \frac{\partial \tilde{h}}{\partial \tilde{x}} & =\frac{1}{1+2 \tilde{h}}, \\
\frac{\tilde{U}_{p}}{1+2 \tilde{h}} & =\frac{\partial \tilde{\sigma}}{\partial \tilde{x}}+A\left(\tilde{\tau}_{s}-\tilde{U}_{p}^{2}\right),
\end{aligned}
$$


where we are assuming that the ice drift is toward the right $\left(\tilde{\tau}_{s}>\tilde{U}_{p}^{2}, \tilde{U}_{p}>0\right)$. The lefthand side of $(4.1 b)$ is the drag caused by the fact that ice is assumed to be motionless when it forms. This drag term is typically much smaller than the other terms in the equation, and could therefore be neglected, but we retain it here for completeness, although we assume below for convenience that $0<\tilde{U}_{p} /(1+2 \tilde{h})<A\left(\tilde{\tau}_{s}-\tilde{U}_{p}^{2}\right)$ is always the case. We also expect $\tilde{h}$ to be a monotonically increasing function of $\tilde{x}$, so that we can use $\partial / \partial \tilde{x}=\partial \tilde{h} / \partial \tilde{x} \partial / \partial \tilde{h}$ in combination with (4.1a) to rewrite $(4.1 b)$ as

$$
-\frac{\partial \tilde{\sigma}}{\partial \tilde{h}}=A\left(\tilde{\tau}_{s}-\tilde{U}_{p}^{2}\right) \tilde{U}_{p}(1+2 \tilde{h})-\tilde{U}_{p}^{2}
$$

In what follows, we will use this expression to characterise the solutions of (4.1) and examine their dependence on the rheological formulation. We will also show how the existence and stability of such solutions are intimately related to the asymptotic properties of the steady-state solutions to (2.11) for large $\tilde{x}$. A note of warning is apposite here: $\S \S 4.1 .1$ and 4.1.2 below are difficult to follow, not so much for the mathematics, which are fairly simple, as for the large number of cases and solution behaviours encountered and there discussed. Some readers might wish to skip the intermediate analysis and developments and refer directly to the summary provided in $\S 4.1 .3$.

\subsubsection{Hydrostatic pressure case}

In the hydrostatic pressure case, $-\partial \tilde{\sigma} / \partial \tilde{h}=2 \tilde{h}$, and (4.2) can only be satisfied for a particular critical ice thickness, namely,

$$
\tilde{h}_{c r i t}=\frac{A\left(\tilde{\tau}_{s}-\tilde{U}_{p}^{2}\right)-\tilde{U}_{p}}{2\left(1 / \tilde{U}_{p}-A\left(\tilde{\tau}_{s}-\tilde{U}_{p}^{2}\right)\right)},
$$

rather than for an infinite thickness range, as required by $(4.1 a)$. It is not possible therefore for the new-ice region to move as a rigid-body in this case. And so we conclude that a bona fide steady-state polynya solution (i.e. one consisting of welldefined thin-ice and pile-up regions of finite width upstream of a new-ice region that extends arbitrarily far offshore) does not exist for the hydrostatic pressure formulation.

It is nevertheless worthwhile to analyse (4.2) more closely to try to gain further insight into the polynya dynamics in the hydrostatic pressure case. For this purpose, we have simultaneously plotted in figure 3(a) the right-hand side of (4.2) (dash-dotted lines) and the left-hand side of the same expression, which, in this case, is $2 \tilde{h}$ (solid line). The values of $A, \tilde{\tau}_{s}$ and $\tilde{U}_{p}$ in these examples are the same that have been used for the calculation of the numerical polynya solutions presented later in $\S 4.2$ with $\tilde{U}_{p}=0.053\left(U_{p}=0.02 \mathrm{~m} \mathrm{~s}^{-1}\right)$ and either $F_{0}=0.72 \mathrm{~m} \mathrm{day}^{-1}$ or $F_{0}=0.12 \mathrm{~m} \mathrm{day}^{-1}$. When the slope of the solid curve is larger than the slope of the dash-dotted curve, the two curves intersect at $\tilde{h}=\tilde{h}_{\text {crit }} \geqslant 0$. If the ice were capable of attaining rigidbody motion with velocity $\tilde{U}_{p}$ in some region where $\tilde{h}>\tilde{h}_{\text {crit }}$, the pressure gradient in the system would be greater than that required by the balance (4.2) to maintain the state of rigid-body motion, and so the ice would need to decelerate eventually. In contrast, if the solid line in figure 3(a) lies always below the dash-dotted line, then the back pressure gradient in any region where rigid motion with velocity $\tilde{U}_{p}$ occurs would be insufficient to balance the right-hand side of (4.2), and the ice would have ultimately to accelerate. In either case, sustained rigid motion with velocity $\tilde{U}_{p}$ will not be possible. 


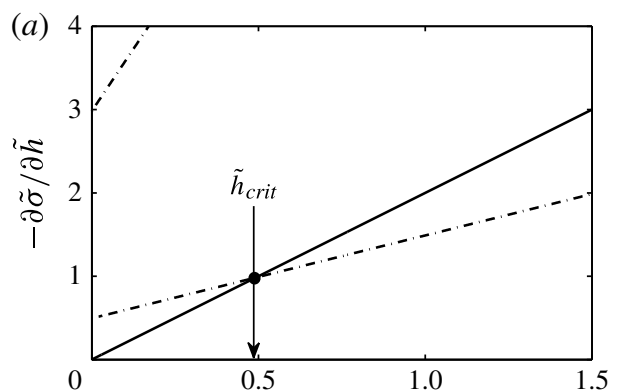

(b)
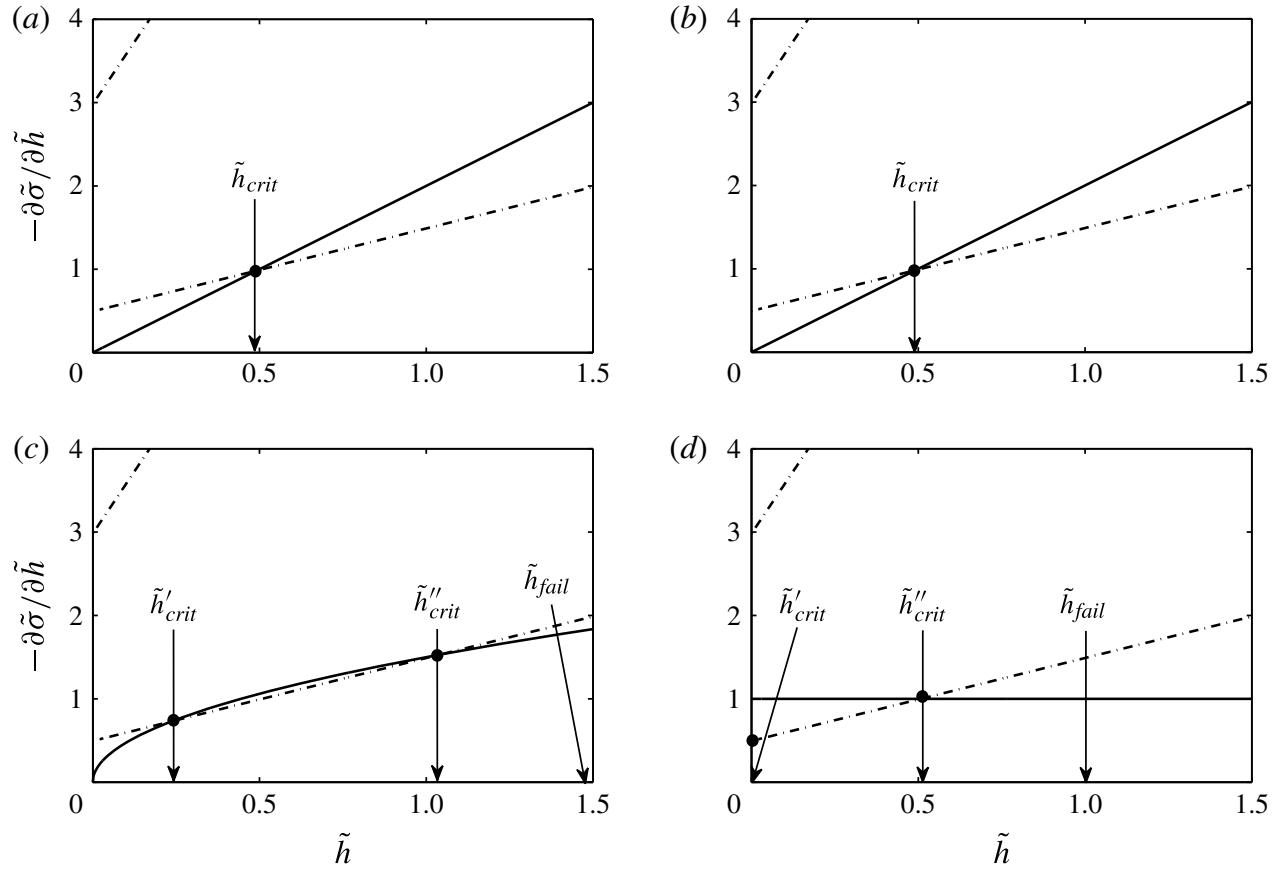

Figure 3. Polynya dynamics. (a) Hydrostatic case, where the solid curve represents $-\partial \tilde{\sigma} / \partial \tilde{h}=2 \tilde{h}$, and the two dash-dotted curves represent $-\partial \tilde{\sigma} / \partial \tilde{h}$ as a function of $\tilde{h}$ according to (4.2) for the values of $A, \tilde{\tau}_{s}$ and $\tilde{U}_{p}$ quoted in $\$ 4.1 .1$. The dash-dotted curve passing across the top left corner of the panel is obtained for a value of $F_{0}$ of $0.12 \mathrm{~m} \mathrm{day}^{-1}$ and the other dash-dotted curve is for $F_{0}=0.72 \mathrm{~m} \mathrm{day}^{-1}$. The intersection between the solid and dash-dotted lines is marked by a small black circle, and occurs at $\tilde{h}=\tilde{h}_{\text {crit }}$. Panels $(b),(c)$ and $(d)$ are similar to $(a)$, but the solid curve represents $-\partial \tilde{\sigma}_{\min } / \partial \tilde{h}=n \tilde{h}^{n-1}$ for the plastic cases $n=2, n=3 / 2$ and $n=1$, respectively. The possible multiple values of $h_{\text {crit }}$ are marked, and also indicated, when pertinent, is the thickness, $\tilde{h}_{\text {fail }}$, at which ice fails (see $\S 4.1 .2$ ). Note that the solid curve in the case $n=1$ is the limit case when $n \rightarrow 1$ of $h=n \tilde{h}^{n-1}$. In the first quadrant, this curve is made of the horizontal semi-infinite line segment $-\partial \tilde{\sigma}_{\min } / \partial \tilde{h}=1$ and the vertical line segment defined by $\tilde{h}=0$ and $-\partial \tilde{\sigma} / \partial \tilde{h} \leqslant 1$.

We have just seen that steady-state solutions with a thin-ice region of finite width do not exist in the hydrostatic pressure case. What, then, is the behaviour of the system as $\tilde{t} \rightarrow \infty$ ? This question is addressed next. We shall see that the asymptotic solution of (2.11) for large $\tilde{t}$ and $\tilde{x}$ sheds, in fact, further light on the problem of determining the polynya's transient evolution.

Let us assume that a steady state can be approached as $\tilde{t} \rightarrow \infty$. It is then permissible to ignore all time derivatives in (2.11). It seems also reasonable to postulate that $\tilde{u}$ will asymptote to a uniform value as $\tilde{x} \rightarrow \infty$. This value, which we denote by $\tilde{u}_{\infty}$, must be bound between 0 and $\sqrt{\tilde{\tau}_{s}}$, the free-drift speed of ice. It is not difficult to find from (2.11a) that, in the steady state, $\tilde{h} \sim \sqrt{\tilde{x} / \tilde{u}_{\infty}}$ as $\tilde{x} \rightarrow \infty$. Introducing this asymptotic behaviour in $(2.11 b)$, we obtain $\partial(\tilde{h} \tilde{u} \tilde{u}) / \partial \tilde{x} \rightarrow 0, \partial(\tilde{h} \tilde{M} \partial \tilde{u} / \partial \tilde{x}) / \partial \tilde{x} \rightarrow 0$, and $\partial \tilde{\sigma} / \partial \tilde{x} \rightarrow$ $-1 / \tilde{u}_{\infty}$ in the limit when $\tilde{x} \rightarrow \infty$. An asymptotic momentum balance is therefore established between pressure gradient, wind stress and basal friction. Therefore, the 
asymptotic form of (2.11b) turns out to be such that $\tilde{u}_{\infty}$ is a solution of the equation: $\mathcal{F}(\tilde{u}) \stackrel{\text { Def }}{=}-1 / \tilde{u}+A\left(\tilde{\tau}_{s}-\tilde{u}^{2}\right)=0$. If $\eta \stackrel{\text { Def }}{=} 1 /\left(A \tilde{\tau}_{s}^{3 / 2}\right) \leqslant 2 / 3^{3 / 2}$, then $\mathcal{F}(\tilde{u})$ has three real roots, one of them negative and the other two positive. In contrast, if $\eta>2 / 3^{3 / 2}$, the negative real root of $\mathcal{F}(\tilde{u})$ remains, but the other two roots acquire imaginary parts. To first order in $\eta$, the positive real roots of $\mathcal{F}(\tilde{u})$ can be approximated by $\tilde{u}_{\infty}^{\prime}=\tilde{\tau}_{s}^{1 / 2} \eta$ and $\tilde{u}_{\infty}^{\prime \prime}=\tilde{\tau}_{s}^{1 / 2}(1-1 / 2 \eta)$. They can become arbitrarily close to the limiting values 0 and $\tilde{\tau}_{s}^{1 / 2}$ (i.e. free drift), respectively, for large enough values of $A$ or $\tilde{\tau}_{s}$.

It may strike the reader as somewhat odd that two positive asymptotic solutions can exist for this problem, and it is natural to speculate that only one of them may be stable. Let us denote the two positive roots of $\mathcal{F}(\tilde{u})$, if they exist, by $\tilde{u}_{\infty}^{\prime}$ and $\tilde{u}_{\infty}^{\prime \prime}$, with $\tilde{u}_{\infty}^{\prime} \leqslant \tilde{u}_{\infty}^{\prime \prime}$. All of the solutions of the hydrostatic case that we have calculated numerically are such that the ice velocity asymptotes to the largest of these two velocities, which prompts us to conjecture that, of the two roots of $\mathcal{F}(\tilde{u})$, only $\tilde{u}_{\infty}^{\prime \prime}$ corresponds to an asymptotically stable solution. The following heuristic argument supports this conjecture. Consider a steady-state solution of (2.11) for values of $A$ and $\tilde{\tau}_{s}$ such that positive asymptotic velocities exist. The curve $\mathcal{F}(\tilde{u})$ for such a case is shown in figure 4. Assume now that a small, constant and uniform, impulsive perturbation, $\Delta \tilde{\tau}_{s}$, is added to the wind stress. (For simplicity, we perturb $\tilde{\tau}_{s}$ but not $A$. However, our argument can be easily generalised to the case when both $\tilde{\tau}_{s}$ and $A$ are given impulsive changes.) As a result of the perturbation, the asymptotic steady-state velocities will change by the small amounts $\Delta \tilde{u}_{\infty}^{\prime}$ and $\Delta \tilde{u}_{\infty}^{\prime \prime}$, as shown in figure 4. It is evident from the figure that the sign of $\Delta \tilde{u}_{\infty}^{\prime}$ is opposite to that of $\Delta \tau_{s}$, while the signs of $\Delta \tilde{u}_{\infty}^{\prime \prime}$ and $\Delta \tau_{s}$ covary. In other words, to reach a new steady state after the application of a positive (negative) wind perturbation, $\Delta \tau_{s}$, requires a decrease (increase) in the asymptotic velocity if the initial state had $\tilde{u}_{\infty}=\tilde{u}_{\infty}^{\prime}$, which is physically implausible. In contrast, if $\tilde{u}_{\infty}=\tilde{u}_{\infty}^{\prime \prime}$, the required change in the asymptotic velocity is consistent with the one that would be dynamically expected from the change in the wind.

Obviously, when positive real roots of $\mathcal{F}(\tilde{u})$ do not exist, no steady-state solution of (2.11) is possible. How does the unsteady solution look in this case as $\tilde{t} \rightarrow \infty$ ? An instance of an unsteady solution will be presented in the next section, but we can here argue in general that, since an asymptotic value of the ice velocity must exist and it cannot be positive, then it must be zero, with an asymptotic momentum balance dominated by only two terms, namely, the wind stress and the pressure gradient force. It is obvious that, for motionless ice, the mass conservation equation (2.11a) does not permit a steady-state solution, and so the ice must grow thermodynamically to arbitrarily large thickness.

The asymptotic properties of the solutions to (2.11) for large $\tilde{t}$ are directly linked to the polynya behaviour discussed at the beginning of this section. Mathematically, this is apparent from the fact that the denominator of (4.3) is proportional to $-\mathcal{F}\left(\tilde{U}_{P}\right)$. When $\mathcal{F}(\tilde{u})$ has no positive real roots, $\mathcal{F}(\tilde{u})$ is negative for all $u$, and $h_{\text {crit }}>0$ for all $\tilde{U}_{p}$. Noting that, as the polynya opens, the ice thickness will first attain the critical value $\tilde{h}_{c r i t}$ at $\tilde{x}=\tilde{X}_{p}$ and that, as long as horizontal viscosity is neglected, equation $(4.1 b)$ is exact at $\tilde{x}=\tilde{X}_{p}$, it is easy to see that excess back pressure in the new-ice region will start to build up at some point around the time when the ice thickness reaches its critical value at $\tilde{x}=\tilde{X}_{p}$, thus forcing the ice to gradually slow down. This behaviour will be confirmed numerically in $\S 4.2$.

When positive real roots of $\mathcal{F}(\tilde{u})$ exist, they separate the values of $\tilde{U}_{p}$ for which $\tilde{h}_{c r i t}$ is positive or negative. For $0 \leqslant \tilde{U}_{p} \leqslant \tilde{u}_{\infty}^{\prime}, \mathcal{F}\left(\tilde{U}_{p}\right)$ is negative, $\tilde{h}_{c r i t}$ is positive, and, as explained in the previous paragraph, back pressure in the new-ice region will 


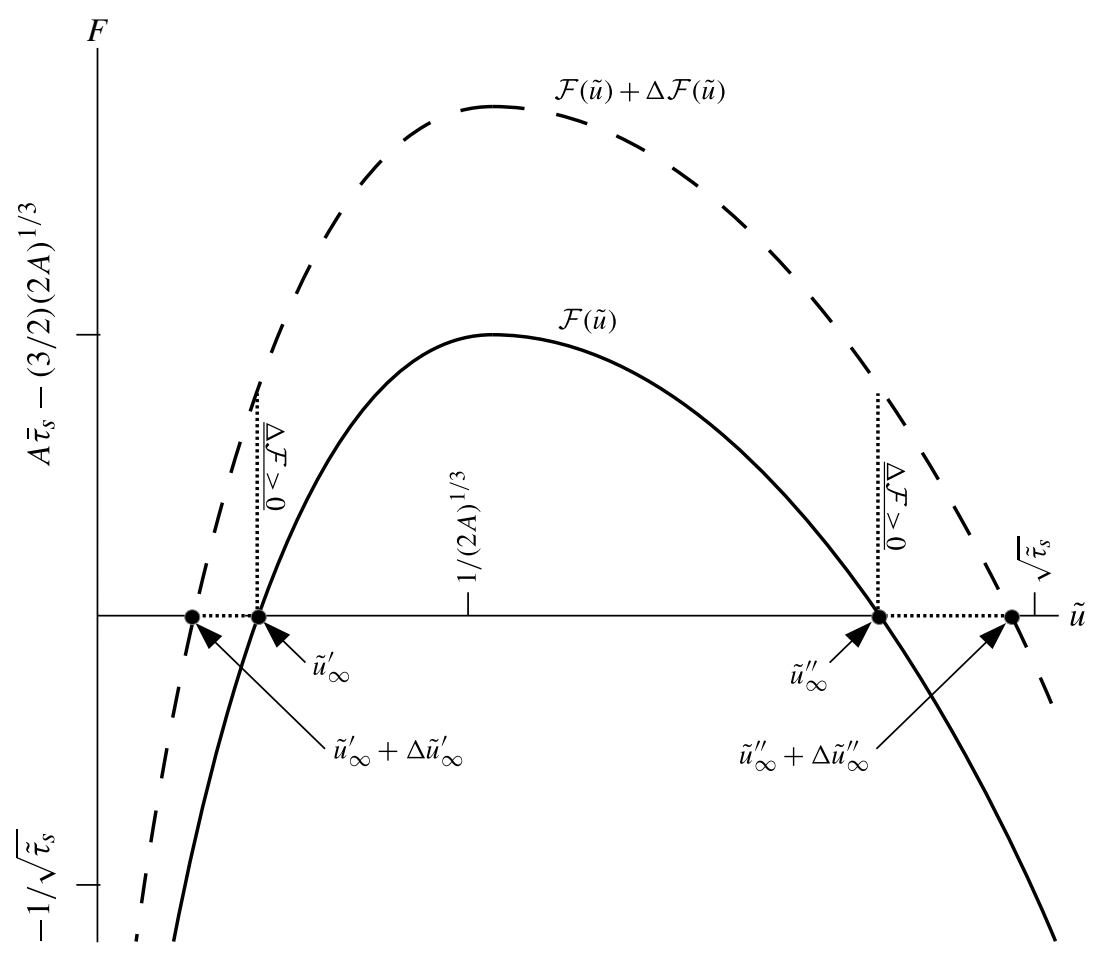

FIGURE 4. Hydrostatic pressure case. The solid line represents the curve $\mathcal{F}(\tilde{u})$ for values of $A$ and $\tilde{\tau}_{s}$ such that two positive real asymptotic velocities, $\tilde{u}_{\infty}^{\prime}$ and $\tilde{u}_{\infty}^{\prime \prime}$, exist. For reference, the free-drift velocity, $\sqrt{\tilde{\tau}_{s}}$, and the corresponding value of $\mathcal{F}(\tilde{u})$, namely, $-1 / \sqrt{\tilde{\tau}_{s}}$, are indicated in the figure. The maximum value of $\mathcal{F}(\tilde{u})$ is $A \tilde{\tau}_{s}-3 / 2(2 A)^{1 / 3}$ and occurs at $\tilde{u}=(2 A)^{-1 / 3}$. Consequently, $\tilde{u}_{\infty}^{\prime \prime} \geqslant(2 A)^{-1 / 3}$ and $\tilde{u}_{\infty}^{\prime} \leqslant(2 A)^{-1 / 3}$. The dashed line represents the curve $\mathcal{F}(\tilde{u})+\Delta \mathcal{F}(\tilde{u})$ that results from applying a small impulsive perturbation $\Delta \tilde{\tau}_{s}$ to the wind stress. As a result of the change in $\tilde{\tau}_{s}$, the asymptotic steady-state velocities change by $\Delta \tilde{u}_{\infty}^{\prime}$ and $\Delta \tilde{u}_{\infty}^{\prime \prime}$, the former change being of opposite sign to $\Delta \tilde{\tau}_{s}$ while the latter has the same sign as $\Delta \tilde{\tau}_{s}$.

eventually force the ice velocity to drop to zero. If $\tilde{u}_{\infty}^{\prime}<\tilde{U}_{p}<\tilde{u}_{\infty}^{\prime \prime}, \mathcal{F}\left(\tilde{U}_{p}\right)$ is positive, $\tilde{h}_{c r i t}$ is negative and the pressure gradient in the new-ice region will be unable to balance the combined wind and bottom friction stress. The new-ice region will therefore recede downwind, leaving behind an increasingly widening thin-ice region. The ice velocity in the thin-ice region will asymptote toward $u_{\infty}=\tilde{u}_{\infty}^{\prime \prime}$. Finally, if $\tilde{u}_{\infty}^{\prime \prime}<\tilde{U}_{p}, \mathcal{F}\left(\tilde{U}_{p}\right)$ is again negative, and $\tilde{h}_{c r i t}$ positive. However, in this case, when the critical thickness is attained at $\tilde{x}=\tilde{X}_{p}$, the excess back pressure will force the ice velocity to drop toward the stable steady-state asymptotic ice velocity $u_{\infty}=\tilde{u}_{\infty}^{\prime \prime}$, rather than toward zero. The thin-ice region of the polynya will again become arbitrarily wide with time. This behaviour will also be confirmed in the numerical simulations presented in $\S 4.2$.

\subsubsection{Plastic case}

In the case of a plastic rheology, $\tilde{\sigma}$ is a single-valued function of $\tilde{h}$ only when the system is in a state of non-zero convergence or divergence. When the system is 
moving as a rigid body, all that can be said with certainty about $\tilde{\sigma}$ is that $\tilde{h}^{n} \geqslant-\tilde{\sigma}$. To aid the following discussion, let us refer to the polynya schematic in figure 1 and assume, for simplicity, that the entire new-ice region is moving rigidly with velocity $\tilde{U}_{p}$. Upstream in the pile-up region, the ice is converging, and therefore $\tilde{h}^{n}=-\tilde{\sigma}$ there. Assuming that both $\tilde{h}$ and $\partial \sigma \tilde{\partial} \tilde{\partial} \tilde{h}$ are continuous at $\tilde{x}=\tilde{X}+\tilde{\Delta}$, we can then use (4.2) to derive an expression for the ice thickness, $\tilde{h}_{c r i t}=\left.\tilde{h}\right|_{\tilde{X}=\tilde{X}+\tilde{X}}$, at the boundary between the pile-up region, where ice is converging, and the new-ice region, where ice is in rigid motion:

$$
n \tilde{h}_{c r i t}^{n-1}-A\left(\tilde{\tau}_{s}-\tilde{U}_{p}^{2}\right) \tilde{U}_{p} 2 \tilde{h}_{c r i t}=A\left(\tilde{\tau}_{s}-\tilde{U}_{p}^{2}\right) \tilde{U}_{p}-\tilde{U}_{p}^{2},
$$

which is equivalent to (4.3) when $n=2$. Geometrically, the solutions to (4.4) are the abscissas of the intersection points between the curves $n \tilde{h}^{n-1}$ and $A\left(\tilde{\tau}_{s}-\tilde{U}_{p}^{2}\right) \tilde{U}_{p}$ $(1+2 \tilde{h})-\tilde{U}_{p}^{2}$, as depicted in figure 3 .

When non-negative solutions of (4.4) do not exist, the new-ice region must recede to $\tilde{x}=\infty$ because, similarly to what was argued in $\S 4.1 .1$, the internal forces are not able to balance the combined wind and bottom stresses at the same time as the ice moves as a rigid-body at speed $\tilde{U}_{p}$. It is straightforward to determine when non-negative roots of (4.4) exist (always under the assumption that $A\left(\tilde{\tau}_{s}-\tilde{U}_{p}^{2}\right)>\tilde{U}_{p}>0$ ). We distinguish the following four cases. First, if $n>2$, there is always one and only one non-negative root (this case is not shown in figure 3). Second, if $n=2$, there will be a single non-negative root if and only if $s=A\left(\tilde{\tau}_{s}-\tilde{U}_{p}^{2}\right) \tilde{U}_{p}<1$. Third, if $1<n<2$, two nonnegative solutions will occur as long as $s-\tilde{U}_{p}^{2} \leqslant n^{1 /(2-n)}(2-n)((n-1) /(2 s))^{(n-1) /(2-n)}$, and none otherwise. Fourth, if $n=1$, two non-negative solutions will exist if and only if $s-\tilde{U}_{p}^{2} \leqslant 1$, one of the solutions being $h_{\text {crit }}=0$.

The existence of non-negative values of $\tilde{h}_{\text {crit }}$ does not guarantee, however, that rigid motion with velocity $\tilde{u}=\tilde{U}_{p}$ will occur downstream of $\tilde{h}=\tilde{h}_{\text {crit }}$. For rigid motion to take place, it is also required that $\tilde{\sigma}_{\min }=-\tilde{h}^{n} \leqslant \tilde{\sigma}$, where $\tilde{\sigma}_{\min }$ is the largest negative internal stress that the ice can sustain. Integrating (4.2) between $\tilde{h}_{\text {crit }}$ and any $\tilde{h}$ downstream of $\tilde{h}_{\text {crit }}$, this condition can be expressed as

$$
\tilde{h}^{n}-\tilde{h}_{c r i t}^{n} \geqslant\left\{A\left(\tilde{\tau}_{s}-\tilde{U}_{p}^{2}\right) \tilde{U}_{p}-\tilde{U}_{p}^{2}\right\}\left(\tilde{h}-\tilde{h}_{c r i t}\right)+A\left(\tilde{\tau}_{s}-\tilde{U}_{p}^{2}\right) \tilde{U}_{p}\left(\tilde{h}^{2}-\tilde{h}_{c r i t}^{2}\right) .
$$

Referring to any of the panels $(b),(c)$ or $(d)$ of figure 3, the left-hand side of (4.5) is the area under the solid curve between $\tilde{h}=\tilde{h}_{\text {crit }}$ and $\tilde{h}>\tilde{h}_{\text {crit }}$. Similarly, the righthand side of (4.5) is the area under the dash-dotted curves between the same two values of the abscissa. If (4.5) holds for all values of $\tilde{h} \geqslant \tilde{h}_{\text {crit }}$, the ice will be able to move as a rigid-body downstream of $\tilde{h}_{\text {crit }}$. However, if (4.5) cannot be satisfied for ice of thickness larger than a threshold value $\tilde{h}=\tilde{h}_{\text {fail }}>\tilde{h}_{\text {crit }}$, then rigid motion is possible for as long as $\tilde{h}_{\text {crit }}<\tilde{h}<\tilde{h}_{\text {fail }}$, but the ice will fail upon reaching the thickness $\tilde{h}_{\text {fail }}$, and rigid motion will not be sustainable beyond that point. For example, when $n=1$ (figure $3 d$ ), $\tilde{h}_{\text {crit }}=0$ is a solution of (4.4) provided that $s-\tilde{U}_{p}^{2} \leqslant 1$. In this case, rigid-body motion with velocity $\tilde{U}_{p}$ is possible, but only until that time when the downstream ice attains a thickness $\tilde{h}=\tilde{h}_{\text {fail }}=\left(1-\left(s-\tilde{U}_{p}^{2}\right)\right) / s$. The inequality (4.5) cannot be satisfied for values of $\tilde{h}>\tilde{h}_{\text {fail }}$. We note that the second value of $\tilde{h}_{\text {crit }}$ that occurs when $n=1$, namely, $\tilde{h}_{\text {crit }}=\left(1-\left(s-\tilde{U}_{p}^{2}\right)\right) /(2 s)$, cannot be the most upstream 
point of a region of rigid motion because (4.5) is not satisfied for any $\tilde{h}$ larger than $\tilde{h}_{c r i t}$ (this is obvious from inspection of figure $3 d$ ). Very similar comments apply to the case $n=3 / 2$ (figure $3 c$ ), and indeed to the entire range $1 \leqslant n<2$. Whenever there are two non-negative roots of (4.4), only the smallest of them is such that rigid motion can be sustained downstream of the point where sea ice attains that critical thickness and up to the point where $\tilde{h}=\tilde{h}_{\text {fail }}$, when the ice will experience compressive failure and rigid motion will cease.

We have just concluded that, when $1 \leqslant n<2$, the thin-ice region of the polynya should, given sufficient time, widen indefinitely, irrespective of whether positive values of $\tilde{h}_{\text {crit }}$ exist or not. We next investigate what are the asymptotic plastic solutions of (2.11) for large $\tilde{t}$ and what do these solutions tell us about the existence, or otherwise, of steady-state polynyas. Assume that a steady state can indeed be achieved as $\tilde{t} \rightarrow \infty$ and that, in this steady state, $\tilde{u}$ tends to a constant value $\tilde{u}_{\infty}$ for $\tilde{x} \rightarrow \infty$. Under these assumptions, equation (2.11a) implies that $\tilde{h} \sim \sqrt{\tilde{x} / \tilde{u}_{\infty}}$ as $\tilde{x} \rightarrow \infty$. And this leads also to $\partial(\tilde{h} \tilde{u} \tilde{u}) / \partial \tilde{x} \rightarrow 0$ and $\partial(\tilde{h} \tilde{M} \partial \tilde{u} / \partial \tilde{x}) / \partial \tilde{x} \rightarrow 0$ in $(2.11 b)$. The asymptotic behaviour of $\partial \tilde{\sigma} / \partial \tilde{x}$ depends on whether $\tilde{u}_{\infty}$ is approached under divergence, rigidly, or under convergence. In the first case, the asymptotic ice internal force would be zero and $\tilde{u}_{\infty}=\sqrt{\tilde{\tau}_{s}}$. In fact, a steady-state solution in which the entire ice cover, from the coast to $\tilde{x}=\infty$, is moving in free drift is obviously always possible in the case of a plastic rheology, irrespective of the value of $n$. In the second case, the asymptotic value of $\partial \tilde{\sigma} / \partial \tilde{x}$ will satisfy $-n /\left(2 \tilde{u}_{\infty}\right)\left(\tilde{x} / \tilde{u}_{\infty}\right)^{(n / 2-1)} \lesssim \partial \tilde{\sigma} / \partial \tilde{x} \lesssim 0$. In the third case, $\partial \tilde{\sigma} / \partial \tilde{x} \sim-n /\left(2 \tilde{u}_{\infty}\right)\left(\tilde{x} / \tilde{u}_{\infty}\right)^{(n / 2-1)}$. For $1 \leqslant n<2$, the asymptotic value of $\partial \tilde{\sigma} / \partial \tilde{x}$ is zero, regardless of the kinematic behaviour of the system as $\tilde{x} \rightarrow \infty$. This means that $\tilde{u}_{\infty}=\sqrt{\tilde{\tau}_{s}}$, and, since free drift is the maximum attainable velocity, it can only be approached under divergence. This result is consistent with the conclusion drawn in the previous paragraph that, given sufficient time, the thin-ice region of the polynya will widen indefinitely if $1 \leqslant n<2$.

For $n>2$, only the asymptotic solutions corresponding to divergent $\left(\tilde{u}_{\infty}=\sqrt{\tilde{\tau}_{s}}\right)$ and rigid $\left(\tilde{u}_{\infty}=\tilde{U}_{p}<\sqrt{\tilde{\tau}_{s}}\right.$ ) ice motion are possible, which is in consonance with the conclusion reached earlier in this section that steady-state polynyas with a thin-ice region of finite width are always attainable when $n>2$.

When $n=2$, the number and type of possible asymptotic solutions depend on whether positive real solutions to $\mathcal{F}(\tilde{u})=0$, where $\mathcal{F}(\tilde{u})$ was introduced in $\S 4.1 .1$, exist. If no such solutions exist, we find ourselves in a case similar to the case $n>2$. Asymptotic states with divergent $\left(\tilde{u}_{\infty}=\sqrt{\tilde{\tau}_{s}}\right)$ and rigid $\left(\tilde{u}_{\infty}=\tilde{U}_{p}<\sqrt{\tilde{\tau}_{s}}\right)$ ice motion are possible and so, therefore, are steady-state polynyas with a thin-ice region of finite width. As we have shown in $\S 4.1 .1$, if positive real solutions to $\mathcal{F}(\tilde{u})=0$ exist, then there are two of them, which we denote by $\tilde{u}_{\infty}^{\prime}$ and $\tilde{u}_{\infty}^{\prime \prime}$. It is then easy to see that the system will asymptotically approach a state of rigid motion with $\tilde{u}_{\infty}=\tilde{U}_{p}$ provided $\tilde{U}_{p}<\tilde{u}_{\infty}^{\prime}$ or $\tilde{u}_{\infty}^{\prime \prime} \leqslant \tilde{U}_{p}$. This is because $\mathcal{F}\left(\tilde{U}_{p}\right)$ is negative in either case, which means that ice internal forces are strong enough to counter the forward push of the combined wind plus ocean stress. Conversely, if $\tilde{u}_{\infty}^{\prime}<\tilde{U}_{p}<\tilde{u}_{\infty}^{\prime \prime}$, the thin-ice region of the polynya will open indefinitely. Using the same line of argumentation outlined in $\S 4.1 .1$, it is possible to show that the asymptotic solution $\tilde{u}_{\infty}=\tilde{u}_{\infty}^{\prime}$ is not stable, and so indefinitely opening polynyas will evolve towards a steady state in which $\tilde{u}_{\infty}=\tilde{u}_{\infty}^{\prime \prime}$.

The results of this section are in stark contrast with those presented in $\S 3$. Steadystate shock model solutions always exist, except for the case $n=1$. In contrast, steadystate polynya solutions in the continuous model are far less common. They never 
occur in the hydrostatic case nor in the plastic rheology case when $1 \leqslant n<2$, although transient quasi-steady states are possible in the latter case. This difference of behaviour between shock and continuous models is due to the very different dynamics of the consolidating and new-ice region, which, in shock and flux models, is assumed to be always moving as a rigid body. In the continuous model, such states of rigid motion are not always attainable and, when this happens, steady-state polynya solutions in which the thin-ice region is of finite width are not possible.

\subsubsection{Summary of existence and properties of steady-state polynya solutions}

For reference and as a guidance for the presentation of numerical solutions in the next section, it is useful to summarise the results of $\S \S 4.1 .1$ and 4.1.2 as follows. (Recall that $\tilde{u}_{\infty}$ denotes the asymptotic ice velocity for large $\tilde{x}, \tilde{h}_{c r i t}$ is the solution of (4.2), and $\tilde{h}_{\text {fail }}$ is the thickness for which ice can no longer sustain rigid motion in the plastic rheology cases.)

(1) Hydrostatic pressure case. This case does not sustain steady-state solutions in which the thin-ice region of the polynya attains a finite, non-zero finite width. Different polynya behaviours can however be observed depending on the value of $\tilde{u}_{\infty}$.

(a) If positive real values of $\tilde{u}_{\infty}$ do not exist, then $\tilde{h}_{c r i t}$ is real and positive irrespective of the value of $\tilde{U}_{p}$, and the thin-ice region will open until a point in time when $\left.\tilde{h}\right|_{\tilde{x}=\tilde{X}_{p}}$ attains the critical value $\tilde{h}_{\text {crit }}$. The thin-ice region will start closing soon afterwards.

(b) If positive real values of $\tilde{u}_{\infty}$ exist, then there are two of them, which we denote by $\tilde{u}_{\infty}^{\prime}$ and $\tilde{u}_{\infty}^{\prime \prime} \geqslant \tilde{u}_{\infty}^{\prime}$, leading to the following three cases.

(i) If $\tilde{U}_{p} \leqslant \tilde{u}_{\infty}^{\prime}$, then the thin-ice region will evolve in a manner similar to that described in (1)(a), opening up to a maximum width and subsequently closing.

(ii) If $\tilde{u}_{\infty}^{\prime}<\tilde{U}_{p} \leqslant \tilde{u}_{\infty}^{\prime \prime}$, the thin-ice region will open indefinitely, with an asymptotic ice velocity of $\tilde{u}_{\infty}^{\prime \prime}$.

(iii) If $\tilde{u}_{\infty}^{\prime \prime}<\tilde{U}_{p}$, the same behaviour as in (1)(b)(ii) will occur.

(2) Plastic rheology cases. Recall that, in these cases, $\tilde{\sigma}=-\tilde{h}^{n}$ in convergence.

(a) $n=2$.

(i) If positive real values of $\tilde{u}_{\infty}$ do not exist, then the thin-ice region will attain a steady-state finite width after $\left.\tilde{h}\right|_{\tilde{x}=\tilde{X}_{p}}$ reaches the critical thickness.

(ii) If positive real values of $\tilde{u}_{\infty}$ exist, then the following cases are possible.

(1) If $\tilde{U}_{p} \leqslant \tilde{u}_{\infty}^{\prime}$, then the polynya behaves as in (2)(a)(i).

(2) If $\tilde{u}_{\infty}^{\prime}<\tilde{U}_{p} \leqslant \tilde{u}_{\infty}^{\prime \prime}$, the thin-ice region opens indefinitely with an asymptotic ice velocity of $\tilde{u}_{\infty}^{\prime \prime}$.

(3) If $\tilde{u}_{\infty}^{\prime \prime}<\tilde{U}_{p}$, the same behaviour as in (2)(a)(ii)(1) will occur.

(b) $1 \leqslant n<2$. In this case, the thin-ice region will, in the long term, end up opening indefinitely, regardless of the forcing, boundary conditions or other parameters. Nevertheless, if $\tilde{h}_{c r i t}$ is real and positive (in which case, there are always two critical thickness values, namely, $\tilde{h}_{c r i t}^{\prime}$ and $\tilde{h}_{\text {crit }}^{\prime \prime}$ ), then the evolution of the system is such that, during the period of time comprised 
between the events $\left.\tilde{h}\right|_{\tilde{x}=\tilde{X}_{p}}=\tilde{h}_{c r i t}^{\prime}$ and $\left.\tilde{h}\right|_{\tilde{x}=\tilde{X}_{p}}=\tilde{h}_{\text {fail }}$, the thin-ice region of the polynya temporarily locks in a quasi-steady state.

(c) $n>2, \tilde{h}_{c r i t}$ is always positive and unique, and the width of the thin-ice region always attains a steady state.

In the following section, we use a numerical model to investigate in some more detail the dynamics of the steady-state and unsteady solutions discussed above.

\subsection{Numerical polynya solutions}

In this section, we seek confirmation of the theoretical results discussed in $\S \S 4.1 .1$ and 4.1.2. To this end, the continuous model equations (2.1)-(2.8) are solved numerically on a staggered grid with a spatial resolution of $\Delta x=1 \mathrm{~m}$, using a conservative finite-volume upstream scheme for (2.1a) (e.g. Morales Maqueda \& Holloway 2006) and a centred-difference formulation for $(2.1 b)$. Although the model results are presented in the non-dimensional framework introduced at the end of $\S 2$, the model itself solves the dimensional version of the equations. The parameters used in the simulations are as follows. The time stepping is Euler forward with a time step, $\Delta t$, of $0.25 \mathrm{~s}$. Ice internal forces are represented using both hydrostatic pressure and plastic rheology formulations. In the latter case, the rheology was solved using the elastic-viscous-plastic scheme of Hunke \& Dukowicz (1997), with $n$ equal to $1,3 / 2$, and 2 , and corresponding $P^{\star}$ values as listed in table 1 . The chosen values for $P^{\star}$ are such that the velocity scale $u_{c}$ is the same for all rheologies. All other parameters used in the model are also listed in table 1.

For each of the four rheology cases (hydrostatic, plastic $n=2$, plastic $n=3 / 2$ and plastic $n=1$ ), we have run two main experiments: one for which there are no non-negative values of $h_{c r i t}$ and one for which non-negative $h_{\text {crit }}$ values exist. In all of these experiments, the wind stress and the consolidated ice velocity were uniform and constant with values of $0.03 \mathrm{~N} \mathrm{~m}^{-2}$ and $0.02 \mathrm{~m} \mathrm{~s}^{-1}$, respectively. Two values for the frazil ice production in open water were used, namely, $0.12 \mathrm{~m} \mathrm{day}^{-1}$ and $0.72 \mathrm{~m} \mathrm{day}^{-1}$. In combination with all of the other chosen parameters, the use of the first of these ice growth rates on the right-hand side of (4.2) leads to the dashdotted curve in the upper left corner of all panels of figure 3 . The second growth rate produces the other dash-dotted line in the panels. According to the theoretical analysis of $\S 4.1$, the $0.12 \mathrm{~m} \mathrm{day}^{-1}$ ice growth rate should invariably lead to polynyas whose thin-ice regions open indefinitely, while the $0.72 \mathrm{~m} \mathrm{day}^{-1}$ rate should generate different types of behaviour depending on the system's rheology. Different experiments had to be run for different lengths of time in order to allow the ice thickness to attain the theoretically calculated critical and failure thicknesses. Table 2 displays these thickness values, non-dimensionalised, when they are real and non-negative. For the hydrostatic pressure case and the plastic rheology case with $n=2$, we have seen that, when positive real values of $u_{\infty}$ exist, the sign of $h_{\text {crit }}$ is not the sole factor controlling the nature of the solution. The value of $U_{p}$ relative to $u_{\infty}^{\prime}$ and $u_{\infty}^{\prime \prime}$ needs also to be considered. For this reason, two additional experiments were carried out for both the hydrostatic pressure and plastic rheology cases with $n=2$ in which $F_{0}=0.12 \mathrm{~m} \mathrm{day}^{-1}$ and either $U_{p}=0.002 \mathrm{~m} \mathrm{~s}^{-1}\left(<u_{\infty}^{\prime}=0.006 \mathrm{~m} \mathrm{~s}^{-1}\right)$ or $U_{p}=0.078 \mathrm{~m} \mathrm{~s}^{-1} \quad\left(>u_{\infty}^{\prime \prime}=\right.$ $\left.0.076 \mathrm{~m} \mathrm{~s}^{-1}\right)$.

In the following, we present the most salient aspects of these numerical simulations, and confirm that the existence and properties of steady-state polynya solutions are consistent with the discussion developed in $\$ 4.1$. The system of equations (2.11) exhibits a remarkable diversity of solutions, a detailed description of which would 


$\begin{array}{lcc}\text { Parameter } & \text { Value } & \text { Units } \\ C_{D} & 5 \times 10^{-3} & - \\ \Delta t & 0.25 & \mathrm{~s} \\ \Delta x & 1 & \mathrm{~m} \\ g & 9.8 & \mathrm{~m} \mathrm{~s}^{-2} \\ M & 1 & \mathrm{~m}^{2} \mathrm{~s}^{-1} \\ \left(n, P^{\star}\right) & (2332.1750) & \left(-, \mathrm{N} \mathrm{m}^{-3}\right) \\ \left(n, P^{\star}\right) & (3 / 2,211.6557) & \left(-, \mathrm{N} \mathrm{m}^{-5 / 2}\right) \\ \left(n, P^{\star}\right) & (1134.8630) & \left(-, \mathrm{N} \mathrm{m}^{-2}\right) \\ \alpha & 1 & -\mathrm{W} \mathrm{m}^{-1} \mathrm{~K}^{-1} \\ \kappa & 2.03 & \mathrm{~W} \mathrm{~m} \mathrm{~K}^{-1} \\ \nu & 10 & \mathrm{~kg} \mathrm{~m}^{-3} \\ \rho_{i} & 950 & \mathrm{~kg} \mathrm{~m}^{-3} \\ \rho_{w} & 1023 & \end{array}$

TABLE 1. Parameter values of the continuous polynya model.

$\begin{array}{lcccc}\text { Rheology } & F_{0}\left(\mathrm{~m} \mathrm{day}^{-1}\right) & \tilde{U}_{p}\left(U_{p}, \text { in } \mathrm{m} \mathrm{s}^{-1}\right) & \tilde{h}_{\text {crit }} & \tilde{h}_{\text {fail }} \\ \text { Hydrostatic/plastic } n=2 & 0.72 & 0.053(0.020) & 0.4922 & - \\ & 0.12 & 0.207(0.078) & 0.3838 & - \\ \text { Plastic } n=3 / 2 & 0.12 & 0.005(0.002) & 0.2354 & - \\ \text { Plastic } n=1 & 0.72 & 0.053(0.020) & 0.2374-1.0413 & 1.4800 \\ & 0.72 & 0.053(0.020) & 0-0.5079 & 1.0159\end{array}$

TABLE 2. Non-dimensional $\tilde{h}_{c r i t}$ and $\tilde{h}_{\text {fail }}$ values as a function of the rheology, $F_{0}$ and $\tilde{U}_{p}$ for the numerical experiments with positive real $\tilde{h}_{c r i t}$.

require an independent article. We will examine the temporal and spatial distributions of the effective ice thickness and ice velocity for each rheological case, but only as far as confirmation of the results of $\S 4.1$ is concerned. A full mechanistic depiction of the evolution of the system will not be attempted. Before embarking in such an analysis, though, it will prove helpful to briefly focus first on a typical solution of the equations and plot some snapshots of $\tilde{h}$ and $\tilde{u}$ to confirm that the model encapsulated in (2.1)-(2.8) can indeed recreate polynya solutions similar to that schematised in figure 1.

Figure 5 shows snapshots of non-dimensionalised frazil ice effective thickness and velocity at regular integration intervals from an initial state before the polynya opened. The case depicted is the hydrostatic pressure one with $F_{0}=0.12 \mathrm{~m}$ day $^{-1}$. The figure's bottom panel clearly reveals a frazil ice distribution qualitatively similar to that depicted in the schematic of figure 1, with a very well-differentiated region of thin ice separated from a region of ice pile-up where the slope of the effective ice thickness is significantly more pronounced. According to the polynya shock model, the non-dimensional steady-state width of the open water region in this case should be $\underline{\tilde{X}} \sim 0.00048$ (or approximately $52 \mathrm{~m}$, which means that the thin-ice region of the polynya implied by the shock model is barely a shore lead), and this steady state should be reached with a non-dimensional e-folding time of $\underline{T} \sim 0.017(\approx 1.3 \mathrm{~h})$. What we see in the continuous model simulation, however, is entirely different: the region 

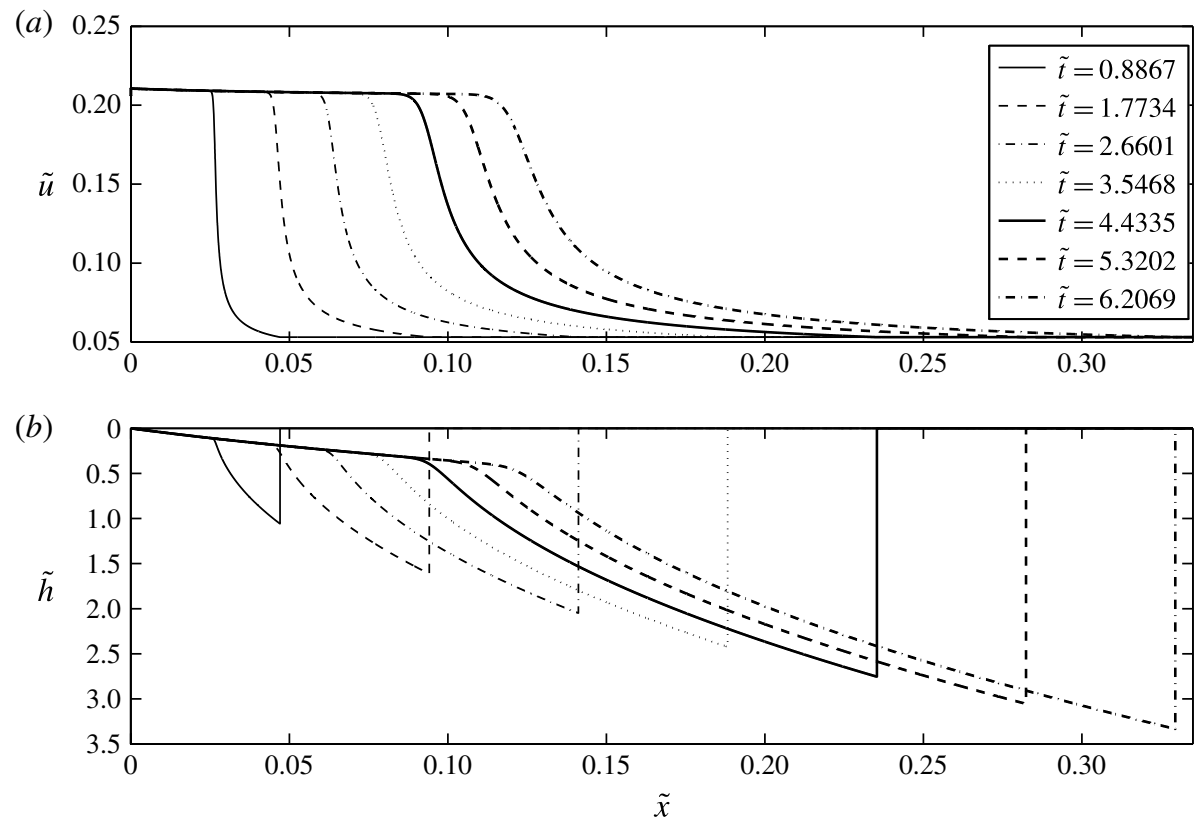

FIGURE 5. Non-dimensional frazil ice velocity (a) and effective frazil ice thickness (b) against non-dimensional distance from the coast simulated with the continuous polynya model in the hydrostatic pressure case with $F_{0}=0.12 \mathrm{~m} \mathrm{day}^{-1}$. The effective frazil ice thickness is set to zero in the pack-ice region, hence the abrupt change in $\tilde{h}$ at the upstream edge of the polynya, which marks the boundary between the new-ice region and the pack ice. The different lines represent $\tilde{u}$ and $\tilde{h}$ at the times indicated in the legend of panel $(a)$.

where the ice moves nearly in free drift is expanding during the whole duration of the integration, which is more than 387 times longer than the $\tilde{T}$ predicted by the shock model. Some degree of deceleration in the opening of the thin-ice region is apparent as time progresses, but there is no indication that a steady state is even close to be achieved by the end of the simulation. This behaviour is consistent with the results of the analysis presented in $\$ 4.1$.

We next present the key results of all of the numerical experiments carried out with the continuous model.

\subsubsection{Hydrostatic pressure case}

Figure 6 shows the evolution of the non-dimensional frazil ice velocity and effective frazil ice thickness in the hydrostatic pressure cases with pack-ice velocity $U_{p}=0.02 \mathrm{~m} \mathrm{~s}^{-1}$ and frazil ice production rates in open water of $F_{0}=0.12 \mathrm{~m} \mathrm{day}^{-1}$ (figure $6 a$ ) and $F_{0}=0.72 \mathrm{~m} \mathrm{day}^{-1}$ (figure $6 b$ ). Figure $6(a)$ is included here for completeness, although it adds little extra information compared with figure 5 , in which ice thickness and velocity profiles from the same experiment are depicted at discrete times. The stable asymptotic value of $\tilde{u}_{\infty}$ is $\sim 0.20$ for this case (to be compared with a free-drift velocity of $\left.\sqrt{\tilde{\tau}_{s}} \approx 0.21\right)$.

Figure $6(b)$ shows results from the case when a positive $\tilde{h}_{\text {crit }}$ exists $(\mathcal{F}(\tilde{u})$ has no positive real roots). According to the discussion of $\S 4.1 .1$, this case leads to polynyas whose thin-ice region opens to a maximum width in a finite time and subsequently 

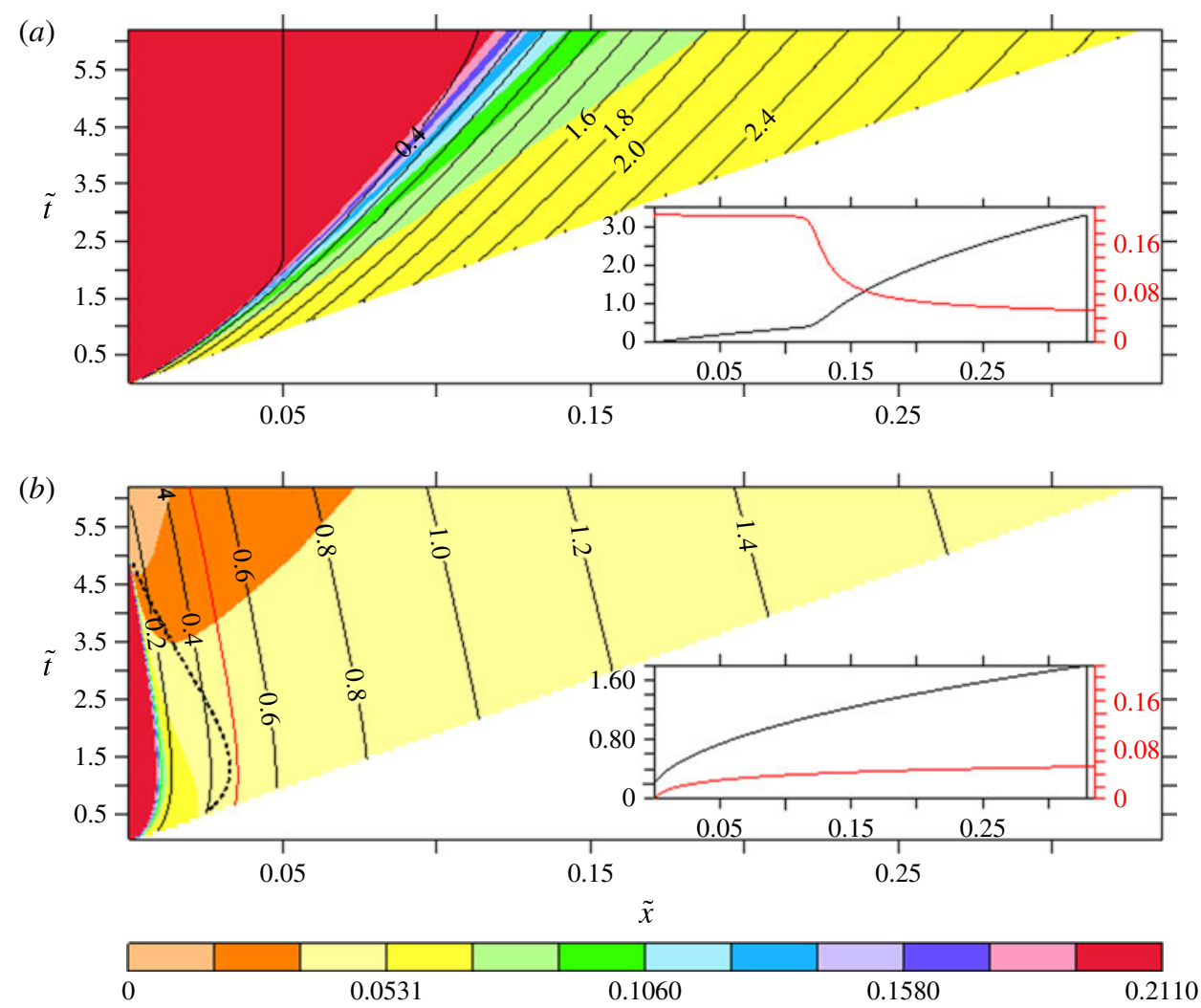

FIGURE 6. Hovmöller diagrams of non-dimensional ice velocity (colour) and ice thickness (contours) for the hydrostatic case integrations with pack-ice velocity of $0.02 \mathrm{~m} \mathrm{~s}^{-1}$ and open water frazil ice production rates of $(a) 0.12 \mathrm{~m} \mathrm{day}^{-1}$ and $(b) 0.72 \mathrm{~m} \mathrm{day}^{-1}$. In $(b)$, the red contour corresponds to the critical thickness $\tilde{h}_{c r i t}=0.4922$, and the dotted black line delineates the contour $\partial \tilde{u} / \partial \tilde{x}=0$. The non-dimensional velocity of the pack ice is $\tilde{U}_{p}=0.0531$. The blanked out area located to the right of the line $\tilde{x}=\tilde{U}_{p} \tilde{t}$ is the pack-ice region. Note that the velocity colour scale is not uniform: the velocity shading interval is 0.0177 for $0 \leqslant \tilde{u} \leqslant \tilde{U}_{p}$ and 0.0175 for $\tilde{U}_{p}<\tilde{u}$. The ice thickness contour interval is 0.2 . The insets in $(a)$ and $(b)$ show the profiles of $\tilde{h}$ (black curve) and $\tilde{u}$ (red curve) against $\tilde{x}$ at time $\tilde{t}=6.21$. Note that the $\tilde{h}$ scale is not the same for both insets and that, contrary to figure $5(a)$, the $\tilde{h}$-axis (left axis) points upwards in order to make the $\tilde{h}$ and $\tilde{u}$ curves more easily distinguishable.

closes. The Hovmöller diagram shown in figure $6(b)$ does indeed evince this type behaviour. Let us, for expediency, characterise the thin-ice region of the polynya as the region where the ice attains velocities of at least $95 \%$ of the free-drift value (which, in our experiments, is 0.2109 ). This region then approximately coincides with the region filled in red in figure 6 . We see that, in figure $6(b)$, the thin-ice region thus defined initially expands up to a maximum width of just under 0.009 , but then starts to shrink until it vanishes at $\tilde{t}=5.1$, at which points the ice velocity at the coast becomes zero. Figure $6(b)$ suggests that the point $(\tilde{x}=0, \tilde{t}=5.1)$ is a singularity for $\tilde{u}$ (i.e. all isotachs radiate from, or collect at, that point). Once the thin-ice region has closed, all of the ice located shoreward of the pack ice moves with velocities below $\tilde{U}_{p}$. From this point 
in time onwards, an increasingly wide region of ice adjacent to the coast becomes effectively motionless, and the ice in this region thickens thermodynamically without bounds. Evidence of this thermodynamic growth is shown in the inset of figure $6(b)$, which depicts the profiles of $\tilde{h}$ and $\tilde{u}$ at $\tilde{t}=6.21$. The ice thickness at $\tilde{x}=0$ is clearly no longer zero. At this time, $\tilde{h}=0.2$, and carries on increasing as the simulation progresses. The width of the thin-ice region starts decreasing near the time when $\tilde{h}\left(\tilde{X}_{p}\right)$ reaches the critical value of $\tilde{h}_{\text {crit }}=0.4922$.

To explore all of the qualitatively different cases discussed in $\S 4.1 .1$, we have also carried out two additional numerical experiments with an open-water ice production of $F_{0}=0.12 \mathrm{~m} \mathrm{day}^{-1}$ but with values of $U_{p}$ below and above $u_{\infty}^{\prime}$ and $u_{\infty}^{\prime \prime}$, respectively. For economy of space, we do not show Hovmöller diagrams for these simulations, but the results confirm our theoretical analysis. When $\tilde{U}_{p}=0.005<\tilde{u}_{\infty}^{\prime}=0.017$, the open-water region expands up to a point when, shortly after the ice thickness at $\tilde{x}=\tilde{X}_{p}$ has attained the critical value of 0.2354 , the back pressure gradient overcomes the net wind plus bottom shear stress, and the thin-ice region starts to close. When $\tilde{U}_{p}=$ $0.207>\tilde{u}_{\infty}^{\prime \prime}=0.202$, the velocity inside the thin-ice region becomes increasingly close to the asymptotic value $\tilde{u}_{\infty}=\tilde{u}_{\infty}^{\prime \prime}$, except near the coast, where $\tilde{u}$ approaches free drift, and in the vicinity of the pack-ice boundary, where $\tilde{u}$ approaches $\tilde{U}_{p}$.

\subsubsection{Plastic case}

Case $n=2$. As we have seen in $\S 4.1 .2$, the plastic case with $n=2$ admits solutions where the polynya's thin-ice region opens indefinitely and solutions where it reaches a steady-state finite width. Figure $7(a)$ shows an example when the thin-ice region opens indefinitely. Since the chosen value of $P^{\star}$ is the same as for the hydrostatic case, this solution is identical to that shown in figure $6(a)$, in spite of the fact that the rheologies are very different from each other. The solutions shown in figures $6(b)$ and $7(b)$ are initially identical too. However, once the thickness of frazil ice at $\tilde{X}_{p}$ has attained the critical thickness $\left(\tilde{h}_{c r i t}=0.4922\right)$, the two solutions start to diverge from each other. While, in the hydrostatic case, the thin-ice region of the polynya eventually closes, as we have seen in $\S 4.2 .1$, in the plastic case, the thin-ice region opens to a steady-state, finite width of $\underline{\tilde{X}} \approx 0.0096$, with the new-ice region moving as a rigid-body with velocity $\tilde{U}_{p}$.

The thin-ice region reaches its steady-state width in a time of about $\underline{T} \approx 1.8$. It is instructive to compare these steady-state width and time scale with those obtained from the shock model by applying (3.10c) and (3.13), respectively, which are $\underline{\tilde{X}} \approx 0.00048$ and $\underline{\tilde{T}} \approx 0.017$, both figures many times smaller than the values derived from the continuous model. The existence of such large discrepancies demonstrates that the polynya shock model is inapt for the representation of the continuous system's dynamics, even in cases when a steady-state polynya solution exists. Inspection of the insets in figure $7(b)$, which depict the solution at $\tilde{t}=6.21$, a time when the thin-ice region of the polynya is already very close to the steady state, reveals why this is so. While there is a rather abrupt horizontal change in $\partial \tilde{h} / \partial \tilde{x}$ and $\partial \tilde{u} / \partial \tilde{x}$ at $\tilde{x} \approx 0.0096$, no clearly defined jumps in either $\tilde{h}$ or $\tilde{u}$ are discernible. Indeed, the width of the region over which the velocity decays from the free-drift value to $\tilde{U}_{p}$ is, in this particular example, comparable to the width of the thin-ice region upstream. The fact that the ice properties change relatively gently across a fairly broad pile-up region invalidates the assumptions behind the jump conditions (3.1), and makes the shock approach inappropriate for the description even of those cases for which a steady-state polynya solution exists. Indeed, for the shock theory to be applicable, the momentum budget 

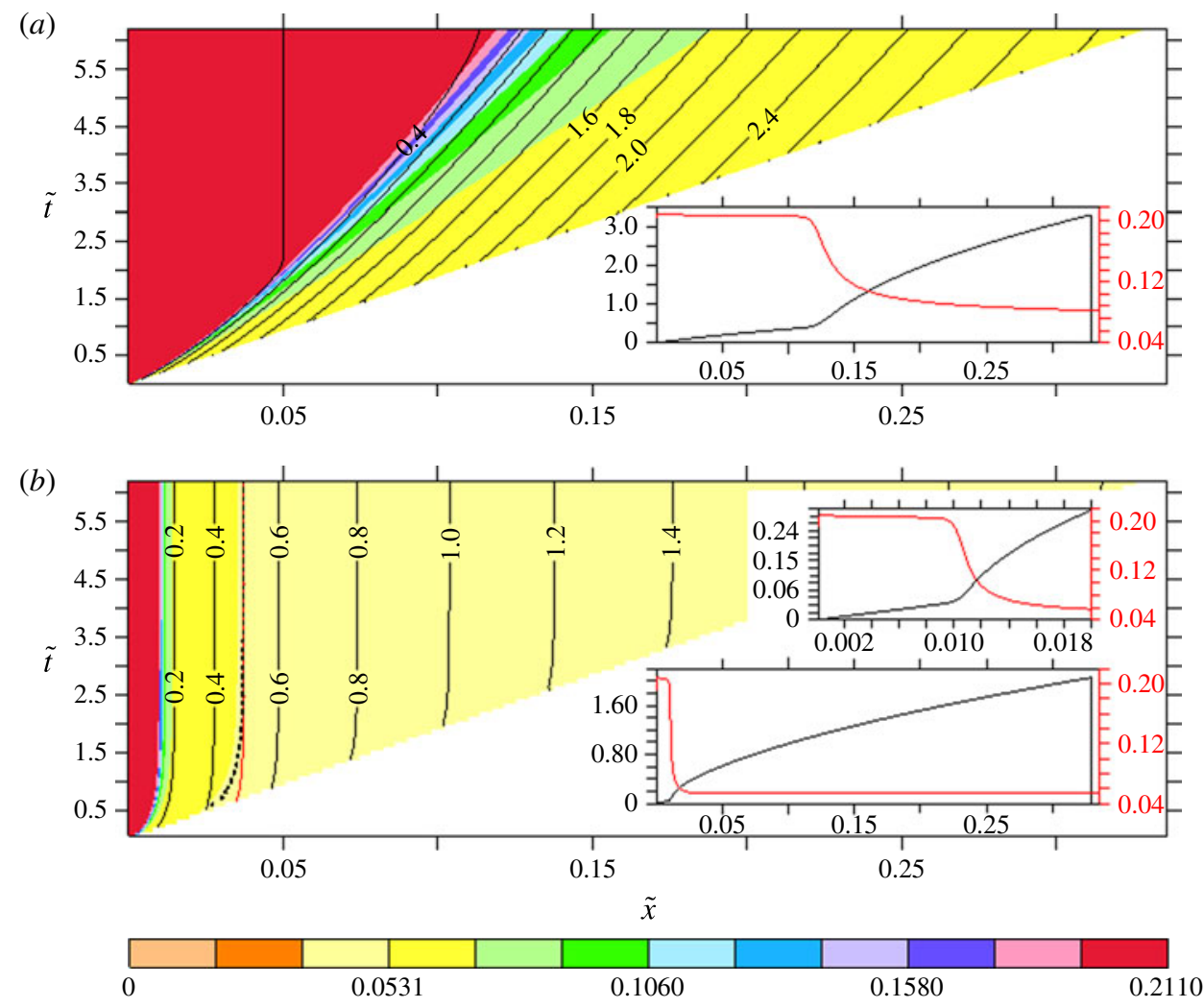

FIgURE 7. As in figure 6 but for the plastic rheology case integrations with $n=2$. The small top inset in panel $(b)$ shows an enlarged view of $\tilde{h}$ and $\tilde{u}$ into the interval $0 \leqslant \tilde{x} \leqslant$ 0.02 .

in the pile-up region needs to be such that the contributions of wind and ocean shear stresses are small compared with those of the internal force and inertial term stresses. However, this is only possible if the pile-up region is sufficiently narrow for $A \int_{\tilde{X}}^{\tilde{X}+\tilde{\Delta}}\left(\tilde{\tau}_{s}-|\tilde{u}| \tilde{u}\right) \mathrm{d} \tilde{x}$ to be much smaller than the change in $\tilde{\sigma}$ and $\tilde{h} \tilde{u}^{2}$ across the region. This is not the case here, as we will further elaborate in $\S 5$.

As in the hydrostatic pressure case, we have performed two more integrations of the plastic rheology case with $n=2, F_{0}=0.12 \mathrm{~m} \mathrm{day}^{-1}$ and values of $U_{p}$ below and above $u_{\infty}^{\prime}$ and $u_{\infty}^{\prime \prime}$, respectively. The results of these two simulations (not shown) validate the conclusions reached in $\S 4.1 .2$. When $\tilde{U}_{p}=0.005<\tilde{u}_{\infty}^{\prime}=0.017$, the thin-ice region opens to a steady state following a transient that coincides with that of the hydrostatic pressure case under the same boundary conditions until the moment when $\left.\tilde{h}\right|_{\tilde{x}=\tilde{X}_{p}} \approx \tilde{h}_{\text {crit }}$. Shortly afterwards, the thin-ice region reaches a stable, steady width rather than closing, as it does in the hydrostatic case. Similarly, when $\tilde{U}_{p}=0.207>$ $\tilde{u}_{\infty}^{\prime \prime}=0.202$, the system evolves initially exactly as it does in the hydrostatic case, but, soon after the effective ice thickness at $\tilde{x}=\tilde{X}_{p}$ reaches the critical value, the new-ice region starts moving rigidly with velocity $\tilde{u}=\tilde{U}_{p}$. However, there is in practice little differentiation between the thin-ice and new-ice regions in this experiment, as both are effectively moving with velocities very close to free drift. 

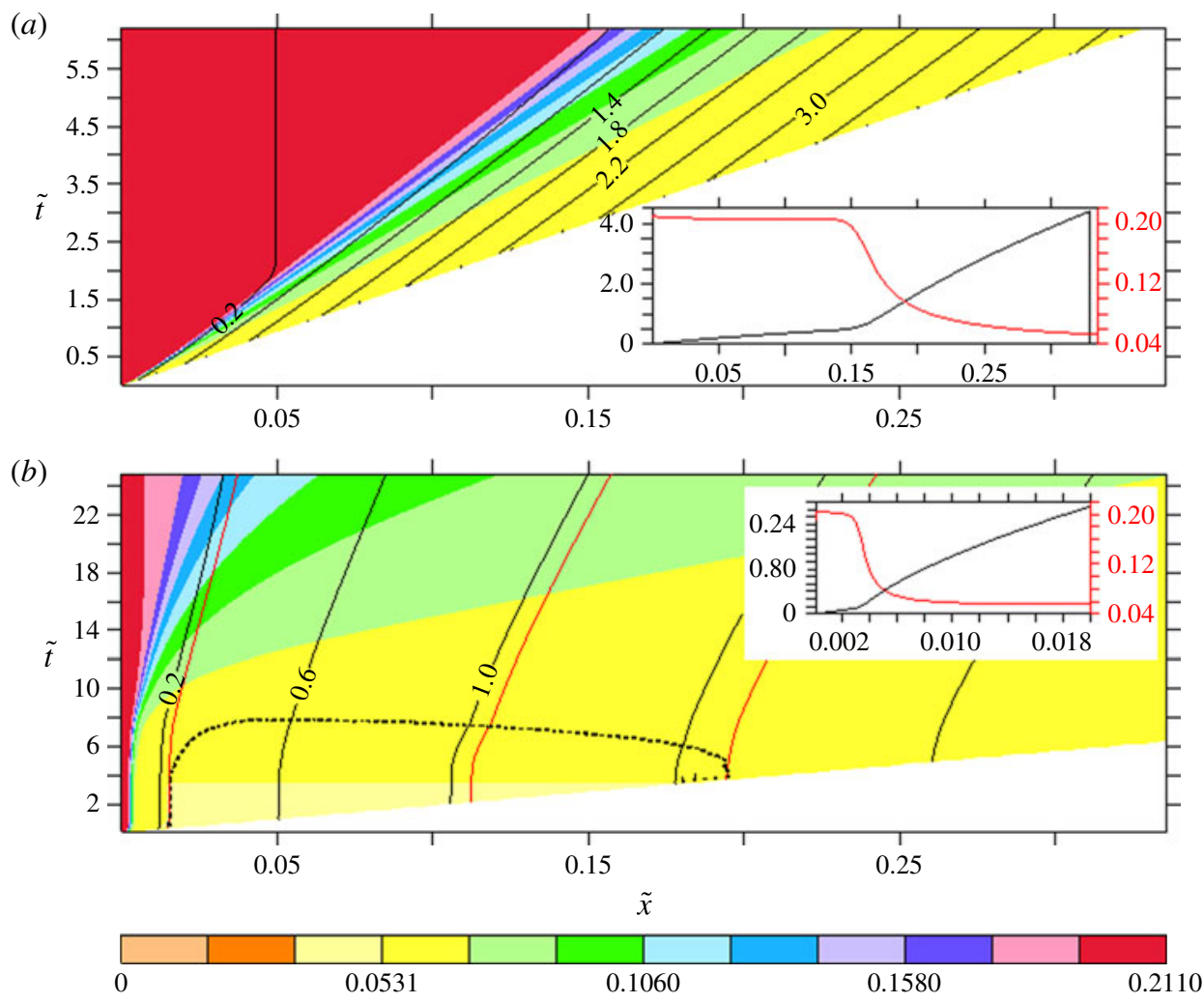

FIGURE 8. As in figure 6 but for the plastic rheology case integrations with $n=3 / 2$. In $(b)$, the red contours correspond to the two critical values of $\tilde{h}\left(\tilde{h}_{\text {crit }}=0.2374\right.$ and $\left.\tilde{h}_{c r i t}=1.0413\right)$ and $\tilde{h}_{\text {fail }}=1.4800$. The ice thickness contour interval is 0.4 . In $(b)$, the abscissa of the inset ranges from 0 to 0.02 only so as to make better discernible the narrow region of thin ice adjacent to the coast.

Case $n=3 / 2$. This case is shown in figure 8 . The case with non-positive $\tilde{h}_{\text {crit }}$ (top panel) requires little comment. The thin-ice region of the polynya opens indefinitely, in accord with the analysis of $\S 4.1 .2$, and the profiles of $\tilde{h}$ and $\tilde{u}$ at any given time are qualitatively similar to the profiles encountered in the hydrostatic and plastic cases when $\tilde{h}_{\text {crit }}$ has no positive real roots.

More interesting is the case when positive real values of $\tilde{h}_{c r i t}$ exist. The polynya evolution in this simulation, which is shown in figure $8(b)$, is also consistent with the discussion presented in $\S 4.1 .2$. A narrow quasi-steady-state polynya is established quite early in the simulation, just about the time when the effective ice thickness at $\tilde{x}=\tilde{X}_{p}$ reaches the smallest of the two positive values of $\tilde{h}_{\text {crit }}$. This occurs at $\tilde{t} \approx$ 0.3 . The width of the thin-ice region then remains stationary until $\tilde{t} \approx 3.5$, with the outer edge of the pile-up region located at $\tilde{x} \approx 0.015$. At $\tilde{t} \approx 3.5$ the effective ice thickness at $\tilde{x}=\tilde{X}_{p}$ attains the value $\tilde{h}_{f a i l}$, and the widths of both the thin-ice and pile-up regions start evolving again, as we had anticipated in $\S 4.1 .2$. For as long as a stationary polynya exists, the ice velocity in the consolidating new-ice region is uniform, constant and equal to $\tilde{U}_{p}$. This is made visually apparent in figure $8(b)$ through the superposition of the curve $\partial \tilde{u} / \partial \tilde{x}=0$, which delimits the region within 
which $\tilde{u}$ is uniform, i.e. the region where ice is moving as a rigid-body. Note in passing, that, some ice is still moving rigidly during the interval $3.5 \lesssim \tilde{t} \lesssim 8$, but it does so at a speed that becomes steadily larger than $\tilde{U}_{p}$.

Case $n=1$. This case has some added interest in that a plastic rheology with maximum ice strength varying linearly with $\tilde{h}$ has been one of the most common choices for the representation of ice internal forces in large-scale sea ice climate models since the publication of the influential paper of Hibler (1979). The simulation with a value of $F_{0}$ of $0.12 \mathrm{~m} \mathrm{day}^{-1}$ leads again to a polynya whose thin-ice region opens indefinitely (figure $9 a$ ). Note that, contrary to what occurs in all other cases so far examined in which $\tilde{h}_{\text {crit }}$ is negative or complex, the ice velocity attains a local maximum (i.e. $\partial \tilde{u} / \partial \tilde{x}=0$ ) inside the thin-ice region, and this maximum is located close to the boundary between the thin-ice and the pile-up regions. The reason for the presence of this maximum is that the ice momentum balance within the thin-ice region is established between the wind-plus-ocean shear stress, on the one hand, and the momentum sink caused by ice formation, on the other hand. The former term of the momentum balance is nearly uniform across the thin-ice region, while the latter decreases steadily as the ice thickness increases (so that the ice growth concurrently decreases). As a result, the velocity of the thin ice diverges slightly as it progresses downwind (and so it experiences no internal forces). However, the ice velocity decays sharply when the ice reaches the pile-up region (inset in figure $9 a$ ), thus creating the mentioned local maximum in $\tilde{u}$. As the thin-ice region expands, this local velocity maximum will gradually move offshore and approach free drift, in agreement with the asymptotic analysis presented in $§ 4.1 .2$.

We comment next on the experiment with $F_{0}=0.72 \mathrm{~m} \mathrm{day}^{-1}$. We have seen in $\S 4.1 .2$ that if real, non-negative values of $\tilde{h}_{\text {crit }}$ exist when $n=1$, then one of these values is always $\tilde{h}_{\text {crit }}=0$. Consistent with this fact, all of the ice formed inside the polynya as the wind starts to blow at $\tilde{t}=0$ should be moving with velocity $\tilde{u}=\tilde{U}_{p}$. This is indeed the case in our numerical simulation, as shown in figure $9(b)$. As the polynya opens, a wedge of newly formed ice moving almost uniformly at the same velocity as the pack develops. It is only when the ice at $\tilde{x}=\tilde{X}_{p}$ attains a thickness equal to $\tilde{h}_{f a i l}$, which happens at approximately $\tilde{t}=2$, that the ice inside the polynya starts to sharply accelerate while continuing to move nearly uniformly upstream of the line $\partial \tilde{u} / \partial \tilde{x}=0$. Downstream of this line, the velocity gently decays towards $\tilde{U}_{p}$. Once this complex initial transient is completed, the evolution of the system will become gradually more similar, in a qualitative sense, to that depicted in panel $(a)$.

\section{Discussion}

As the results presented in the previous section demonstrate, a physical system as simple as that represented by (2.1)-(2.8) is capable of producing polynya solutions that are remarkably complex both in their spatial structure and their temporal behaviour. The evolution of real polynyas is unlikely to be simpler. One of the most striking aspects of the continuous model integrations is that the simulated polynyas evolve in ways that greatly differ from the polynya evolution inferred from flux and shock models. The most notable difference is that polynya flux and shock solutions always attain a steady state with a thin-ice region of finite width, whereas this is not always the case in the presumably more realistic continuous formulations, in which the width of the thin-ice region may reach a steady state, but can also increase indefinitely or fall to zero, depending on the forcing, boundary conditions, 

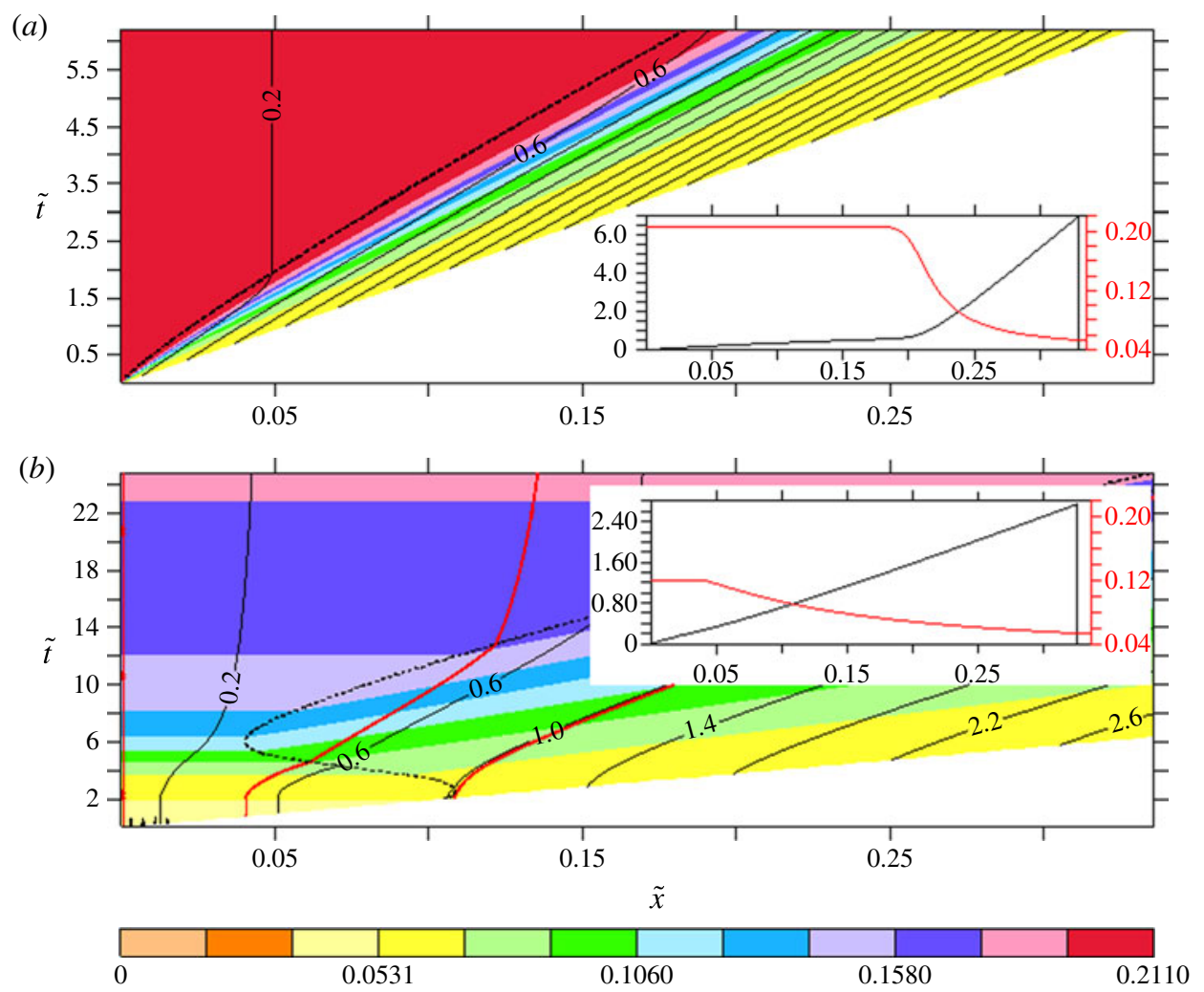

FIGURE 9. As in figure 6 but for the plastic rheology case integrations with $n=1$. In $(b)$, the red contours correspond to the three critical values of $\tilde{h}\left(\tilde{h}_{\text {crit }}=0\right.$ and $\left.\tilde{h}_{\text {crit }}=0.5079\right)$ and $\tilde{h}_{\text {fail }}=1.0159$. The ice thickness contour interval is 0.4 . In both $(a)$ and $(b)$, the dotted black line delineates the points in the $(\tilde{x}, \tilde{t})$ plane where $\partial \tilde{u} / \partial \tilde{x}=0$.

chosen rheology and other model parameters. Even when the continuous model does produce a steady-state polynya of finite width, as, for example, in the plastic case experiment with $n=2$ and $F_{0}=0.72 \mathrm{~m} \mathrm{day}^{-1}$ that we have discussed in the previous section, the solution greatly differs from the one calculated with a shock model.

The object of the following discussion is fivefold. First, we show that, when the effect of the drag caused by frazil ice growth is neglected in the momentum equation, then one single parameter, denoted by $q$ below, suffices to fully determine both $\tilde{h}_{\text {crit }}$ and $h_{\text {fail }}$, the two ice-thickness parameters that characterise the behaviour of the system as far as the existence of polynya steady states and their stability is concerned. Second, we use this result to go back to the dimensional model equations to demonstrate that cases in which polynyas open indefinitely are not exotica of the continuous model, but can in fact occur for perfectly realistic values of the forcing parameters. Third, we show that the reason why shock concepts are not verified by the continuous model is simply that the momentum balance implied by the Rankine-Hugoniot conditions (i.e. momentum advection balanced by hydrostatic pressure or ice internal forces in the pile-up region) is never obtained in the continuous model. Fourth, we put forward a programme for replacing polynya shock models by a more elaborate framework exploiting the different momentum balances predicted by the continuous model in 
the thin-ice, pile-up and new-ice regions. Fifth, we address the issue of whether the use of more complex rheologies in the pile-up region will significantly change our conclusions.

Given the importance of $\tilde{h}_{c r i t}$ in determining the polynya behaviour in the continuous model, it is worthwhile revisiting the relationship between this critical quantity, on the one hand, and the polynya forcing and boundary conditions, on the other hand. According to (4.4), $\tilde{h}_{\text {crit }}$ is a function of $A, \tilde{\tau}_{s}$ and $\tilde{U}_{p}$. The last term on the right-hand side of (4.4) comes about because of the momentum drag caused by the fact that the ice forms at rest. This drag is normally negligible. For example, in the experiments reported in this paper, the ratio between the maximum possible value of the drag term on the left-hand side of $(4.1 b)$, which is the one associated with ice growth, and the ocean drag on the right-hand side of the equation is $1 / A \tilde{U}_{p}$, and this is always smaller than $\sim 0.1$. If, in light of this, we drop the $-\tilde{U}_{p}^{2}$ term from the right-hand side of (4.4) and reorganise the resulting expression, we obtain

$$
q \equiv A\left(\tilde{\tau}_{s}-\tilde{U}_{p}^{2}\right) \tilde{U}_{p}=\frac{n \tilde{h}_{c r i t}^{n-1}}{1+2 \tilde{h}_{c r i t}} .
$$

As a result of this simplification, $\tilde{h}_{\text {crit }}$ becomes an implicit function of the sole parameter $q=A\left(\tilde{\tau}_{s}-\tilde{U}_{p}^{2}\right) \tilde{U}_{p}>0$, which, in turn, depends solely on the forcing $(A$ and $\left.\tilde{\tau}_{s}\right)$ and boundary conditions $\left(\tilde{U}_{p}\right)$. The function $q\left(\tilde{h}_{c r i t}\right)$ given by $(5.1)$ is represented for the three cases $n=2, n=3 / 2$ and $n=1$ in figure 10. Equation (5.1) can be used to approximately determine under what circumstances positive real values of $\tilde{h}_{\text {crit }}$ cannot be attained, in which case, as we have explained above, the thin-ice region of the polynya will steadily widen from the start of the polynya opening and will extend downstream without ever reaching a steady state. We have already seen in $\$ 4.1 .2$ that, if $n>2, \tilde{h}_{\text {crit }}$ is always positive, and this is also immediately obvious from (5.1) (incidentally, positive real values of $\tilde{h}_{\text {crit }}$ always exist as well when $0 \leqslant n<1$ ). When $1 \leqslant n \leqslant 2$, however, $\tilde{h}_{\text {crit }}>0$ only if $0<q \leqslant q_{\max }=\left(n / 2^{n-1}\right)(n-1)^{n-1}(2-n)^{2-n}$. Values of $q$ larger than the upper threshold just quoted lead necessarily to polynyas whose thin-ice region widens indefinitely from the outset. In the plastic case, we note also that, once the momentum drag term associated with ice formation is neglected, the thickness at which ice fails for the first time, $\tilde{h}_{\text {fail }}$, becomes too an implicit function of $q$, namely,

$$
q=A\left(\tilde{\tau}_{s}-\tilde{U}_{p}^{2}\right) \tilde{U}_{p}=\frac{\tilde{h}_{\text {fail }}^{n}-\tilde{h}_{\text {crit }}^{n}}{\tilde{h}_{\text {fail }}-\tilde{h}_{\text {crit }}+\tilde{h}_{\text {fail }}^{2}-\tilde{h}_{\text {crit }}^{2}} .
$$

The previous analysis has been carried out using non-dimensional variables, an approach that is expedient for calculation, but obscures sometimes the interpretation of the physics. We have seen, for example, that, for the plastic rheologies investigated in the paper, the thin-ice region of the polynya will open steadily and indefinitely if positive real values of $\tilde{h}_{\text {crit }}$ do not exist. Using the non-dimensionalised variables, it is difficult to determine whether this behaviour is pathological, perhaps arising only for unrealistic forcing, boundary conditions or model parameters, or whether it occurs commonly. To address this problem, let us express $q$ in dimensional form, i.e.

$$
q=\frac{U_{p}}{F_{0}} \frac{\tau_{s}-\rho_{w} C_{D} U_{p}^{2}}{P^{\star}\left(2 \frac{\kappa}{v}\right)^{n-1}} .
$$

In figure 11, several curves are shown displaying $q=q_{\max }$ for realistic values of $\tau_{s}$, $F_{0}$ and $U_{p}$, and for all four internal force cases that we have been considering in this 


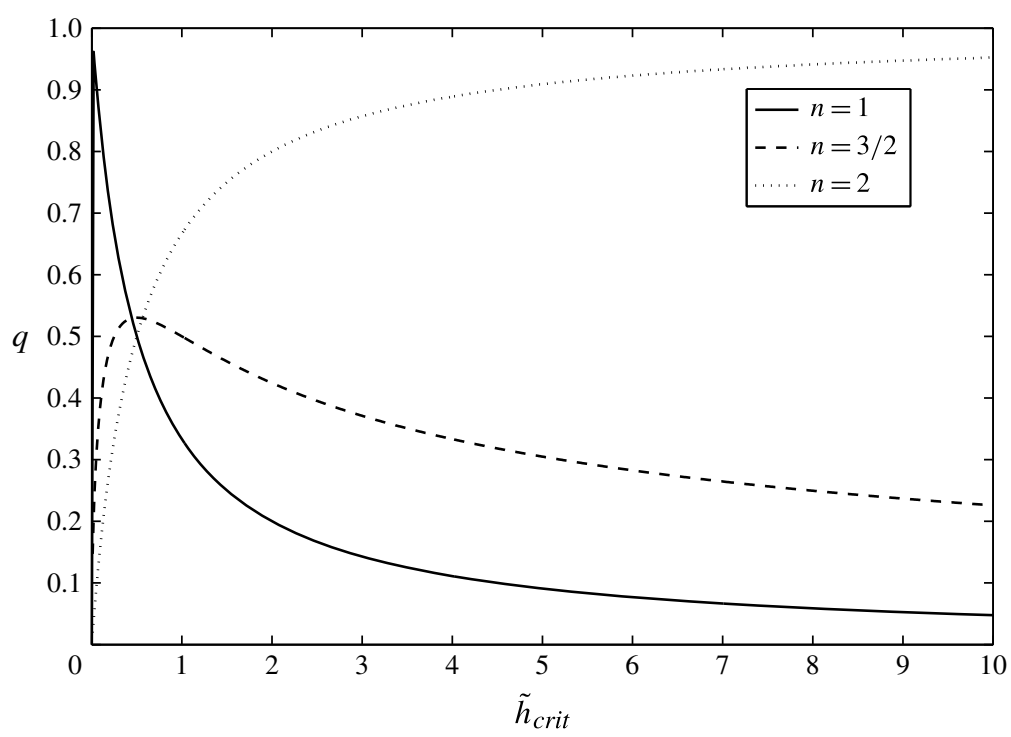

FIGURE 10. Parameter $q=A\left(\tilde{\tau}_{s}-\tilde{U}_{p}^{2}\right) \tilde{U}_{p}$ as a function of $\tilde{h}_{c r i t}$ as given by (5.1) for the three values of $n$ discussed in this paper. Note that there is always a maximum positive $q$ for $\tilde{h}_{\text {crit }}>0$, and therefore values of $q$ larger than the maximum are not compatible with the existence of positive real values of $\tilde{h}_{\text {crit }}$.
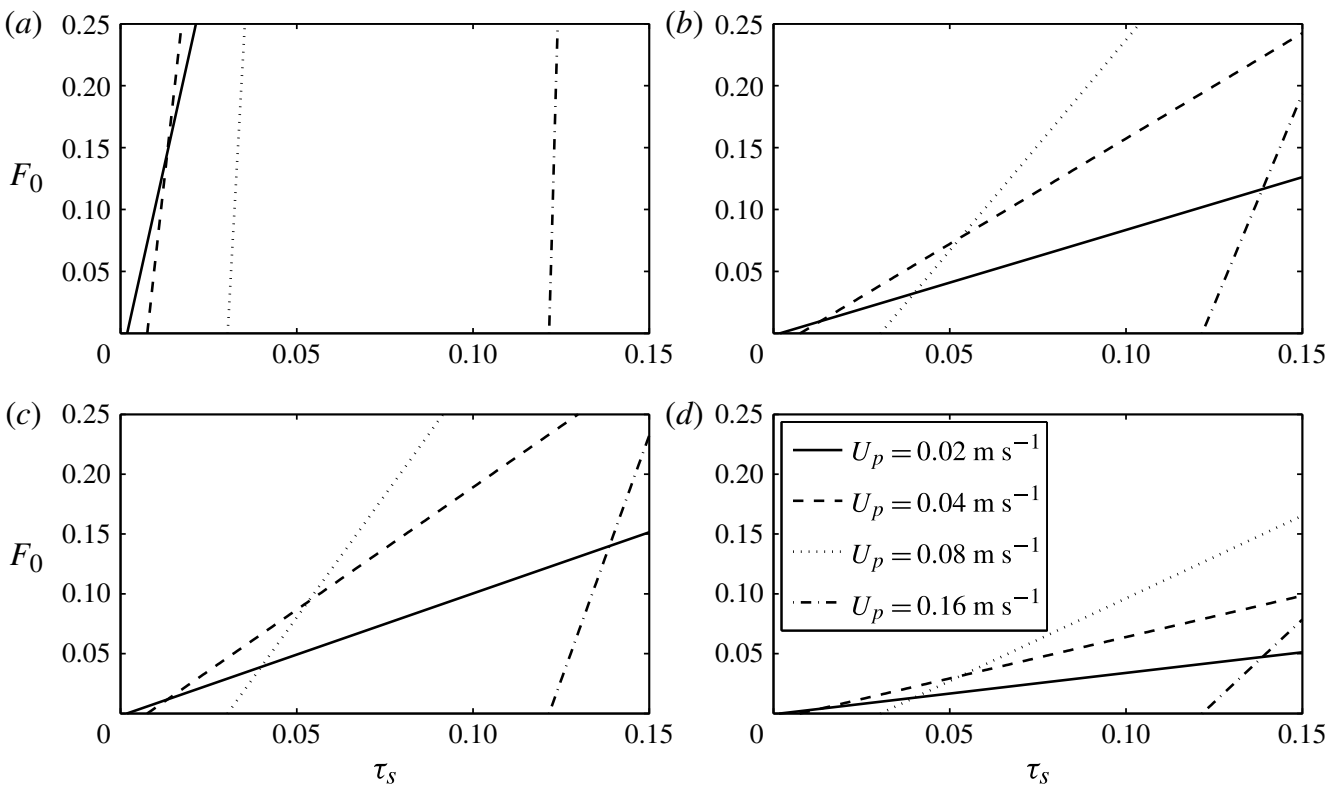

FIGURE 11. Lines of $q=q_{\max }$ for all four rheologies and for realistic ranges of $\tau_{s}$ (in $\mathrm{N} \mathrm{m}^{-2}$ ) and $F_{0}$ (in $\mathrm{m} \mathrm{day}^{-1}$ ). Four different states of uniform pack-ice motion are considered: ( $a$ ) hydrostatic case; $(b)$ plastic case $n=2, P^{*}=5000 \mathrm{~N} \mathrm{~m}^{3} ;(c)$ plastic case $n=3 / 2, P^{*}=5000 \mathrm{~N} \mathrm{~m}^{2.5} ;(d)$ plastic case $n=1, P^{*}=5000 \mathrm{~N} \mathrm{~m}^{2}$.

paper. When the ice rheology is plastic, a $P^{\star}$ value of $5000 \mathrm{~N} \mathrm{~m}^{-(n+1)}$ is chosen, so that the maximum internal force is the same for all cases when $h=1 \mathrm{~m}$. For $n=1$, 
this value of $P^{\star}$ has been used, for example, by Hibler (1979) to parameterise the monthly mean strength of sea ice in the central Arctic. Other constant parameters required for the calculation of $q$ have values as stated in table 1 . In the figure, all points located to the right of a particular curve are such that $q>q_{\max }$, and represent therefore states of motion for which polynyas will open indefinitely. Note that all of the curves intersect the axis of abscissas at $\tau_{s}=\rho_{w} c_{D} U_{p}^{2}$. Points with abscissa smaller than $\rho_{w} c_{D} U_{p}^{2}$ are to be ignored, in accordance to our earlier stated assumption that $\tau_{s} \geqslant \rho_{w} c_{D} U_{p}^{2}$. We note that, irrespective of the rheological case considered, parameter configurations in which the thin-ice region of the polynya widens indefinitely are attainable with realistic forcing, and so this type of polynya evolution cannot be regarded as pathological within the context of the continuous model presented here.

The results of $\S 4$ also show that the progression in time of polynyas simulated by the continuous model is very different from that calculated using flux or shock models. Even when the continuous model produces a steady-state solution, the time scale for reaching such steady state and the steady-state width of the thin-ice region of the polynya are very different from those derived with simpler models. The shock model described in $\S 3$ always leads to steady-state solutions with a finite polynya width which is, in practice, attained in a finite amount of time. In the continuous model, however, only plastic rheologies with $n \geqslant 2$ can produce steady-state polynyas. But, as our plastic experiment with $n=2$ illustrates, even when steady-state solutions exist in the continuous model, the steady-state width of the thin-ice region of the polynya and the time required to reach such equilibrium are very different from those calculated using flux or shock methods. Clearly, the dynamics of the continuous model is not well captured by the more simplistic flux and shock models. In particular, none of the continuous model solutions presented in $\S 4.2$ exhibit well-defined discontinuities in $h$ and $u$, as postulated by flux and shock models. A stable steady-state polynya solution may exist, as, for example, in the case shown in figure 7(b), yet the ice pile-up region cannot be characterised as a shock even in this case. Indeed, for a localised, steep change in velocity and thickness to qualify as a shock, the integral across the shock of the air-ice and ocean-ice stresses (second and third terms on the right-hand side of $(2.11 b)$ ) should be negligible compared with the corresponding integrals for the inertial and internal force terms (left-hand side of $(2.11 b)$ and first term on the right-hand side of the same equation, respectively). This is not the case in the continuous model solutions. If, for argument's sake, we define the pile-up region as the region where the ice velocity decays from $\left.0.95 \tilde{u}\right|_{\tilde{x}=0}$ to $1.05 \tilde{U}_{p}$, it is then easy to confirm from the numerical solution that the leading components of the momentum balance in this region are the internal force term, the wind stress and the ice-ocean shear stress. The integral of $\partial \tilde{\sigma} / \partial \tilde{x}$ across the pile-up region is approximately -0.043 , while the corresponding integrals for the non-dimensional wind and ice-ocean stresses are approximately 0.060 and approximately -0.018 , respectively. The inertial terms are negligible in this region, as they are, in fact, anywhere else in the domain.

Given that the polynya flux and shock theories appear to be inadequate, is there a different set of assumptions that could be used to construct a polynya model, simpler than the continuous formulation, but still capable of reproducing the main features of the polynyas predicted by the continuous model? An alternative to the shock approach is suggested by the fact that the same three-term momentum balance (internal forces, wind stress plus ice-ocean stress) that applies in the pile-up region of the polynya in the continuous model also applies downstream, in the consolidating and new-ice region. This observation can be exploited to devise a reduced physics model in which a free-drift momentum balance would apply in the thin-ice region 
of the polynya, and a 'constrained drift', in which wind and ice-ocean stresses are balanced by the ice internal forces, would apply both in the pile-up and new-ice regions. In combination with the mass conservation equation $(2.11 a)$ and equations guaranteeing the continuity of ice thickness and ice velocity at $(\tilde{x}=\tilde{X})$ the proposed simplified momentum balances in the regions $\tilde{x} \leqslant \tilde{X}$ and $\tilde{X}<\tilde{x}$ can be used to calculate approximate solutions for the distribution of ice thickness and velocity between the coast and the pack ice without having to solve the complete system (2.11). Preliminary tests of this reduced physics approach in the hydrostatic pressure case show that it reproduces remarkably well the time-dependent solutions calculated with the complete continuous model. A full description of the method would lengthen this manuscript excessively, and so we shall leave it for a future manuscript in which a reduced physics model will be used to explore the dependence of simulated polynya length and time scales on forcing fields, boundary conditions and ice and ocean parameters.

A final point, which we have already touched upon in $\S 2$, but is nonetheless worthwhile reiterating here, is that, while the polynya model encapsulated by equations (2.1)-(2.8) may provide a more realistic representation of the dynamics of a polynya than more conventional flux or shock models, it remains nevertheless quite simplistic. This is especially true of its rheology, which, be it hydrostatic or plastic, can only be expected to provide a cartoon-like description of the true ice internal forces. Would our results still hold if more realistic rheologies were substituted for those used in the simulations discussed above? We believe that, qualitatively, the results would indeed endure. To see why, it suffices to consider that, as argued in $\S 2$, a realistic rheology will probably consist of a hydrostatic component in the thin-ice region, where ice is mostly in suspension within the mixed layer, a plastic component for the pack ice and mature ice that may have formed within the polynya, and a transition rheology connecting the previous two through the pile-up region and the shoreward end of the consolidating new-ice region. For such a 'mixed' rheology, the curve of $\partial \tilde{\sigma}_{\min } / \partial \tilde{h}$ against $\tilde{h}$ would be significantly different from the solid curves depicted in figure $3(b)$, (c) or $(d)$ for small values of $\tilde{h}$ (i.e. when the ice is likely to be in suspension in the water column at low volumetric concentrations or have precipitated from the water column onto the ocean surface at small horizontal concentrations) but, for large enough values of $\tilde{h}$, the curves would be similar to those depicted. The parameters for which positive real values of the critical and failure ice thickness exist, and their particular values, would be different from those of the simpler rheologies investigated here. But the main qualitative conclusion that we have reached in this study will remain robust, namely that both steady and unsteady polynya solutions with very different spatial and temporal structures may exist, depending on the forcing, lateral boundary conditions and physical parameters.

\section{Summary and concluding remarks}

We have investigated and elucidated a number of fundamental problems in the theory of polynya dynamics that arise from the use of polynya flux models. Polynya flux models have been used to estimate the contribution of polynyas to salt and dense water production in polar oceans (e.g. Winsor \& Björk 2000; Krumpen et al. 2011). However, although intuitively appealing, the equations underpinning these models have never been derived from first principles. In this paper, we have attempted such a derivation starting from a continuous formulation of the conservation of ice mass and momentum in a polynya, in combination with seemingly reasonable assumptions as to the dominant dynamics operating at the polynya edge. However, the resulting 
flux model, in effect a shock model, does not reproduce well the qualitative, let alone the quantitative, evolution of the polynya calculated using the continuous formulation. Flux/shock model polynyas open to much narrower widths than predicted by the continuous model and do so in much shorter time scales. Flux/shock models fail also to simulate instances, which are not infrequent, in which polynyas do not reach a steady state in the continuous model. This disparity of behaviour between flux and shock models, on the one hand, and continuous models, on the other hand, cast doubts as to the reliability of the former for the investigation of polynya dynamics and for the estimation of haline production and dense water formation in polynyas.

The continuous model introduced in this paper has been used to explore transient and steady-state polynya solutions under a variety of external forcings (wind stress and ice freezing rates), boundary conditions (pack-ice velocity) and rheology formulations (hydrostatic pressure versus plastic internal stresses). A key analytical finding from this model is that, a thin-ice region within which ice moves at near free-drift rates is not necessarily present at all times during the opening of the polynya, and that polynya steady states in which the thin-ice region reaches a finite width are not always attainable. In fact, such solutions only occur for plastic rheologies with ice strength exponent $n \geqslant 2$. A numerical implementation of the continuous model was used to confirm these analytical conclusions and to gain further insight into the properties of transient polynya solutions. A study of the ice momentum balance in the transient polynya solutions demonstrates that the thin-ice region of the polynya is typically in near free drift, and that, in the pile-up and consolidating-ice regions, the momentum balance is between the wind and bottom stresses, on the one hand, and the ice internal forces, on the other hand. This observation suggests possibilities for the formulation of a semi-analytical polynya model, exploiting the fact that different momentum balances apply in different regions of the polynya. This semi-analytical formulation would permit the accurate prediction of the polynya evolution, especially opening length and time scales, without the need to solve numerically the complete set (2.1)-(2.8), which is cumbersome and computationally expensive. Such a reduced-physics model will be presented elsewhere shortly.

\section{Acknowledgements}

This work has been funded by the strategic marine science programme Oceans 2025 of the Natural Environment Research Council, United Kingdom. We thank Professor Kolumban Hutter and three anonymous reviewers for their insightful and constructive comments and recommendations, which have greatly improved this article.

\section{REFERENCES}

Alam, A. \& CURRY, J. 1998 Evolution of new ice and turbulent fluxes over freezing winter leads. J. Geophys. Res. 103, 15783-15802.

BAuer, J. \& MARtin, S. 1983 A model of grease ice growth in small leads. J. Geophys. Res. 88, $2917-2925$.

Biggs, N. R. T., Morales Maqueda, M. A. \& Willmott, A. J. 2000 Polynya flux model solutions incorporating a parameterisation for the collection thickness of consolidated new ice. J. Fluid Mech. 408, 179-204.

De Carolis, G., Olla, P. \& Pignagnoli, L. 2005 Effective viscosity of grease ice in linearized gravity waves. J. Fluid Mech. 535, 369-381.

Doble, M. J., Coon, M. D. \& Wadhams, P. 2003 Pancake ice formation in the Weddell Sea. J. Geophys. Res. 108, 3209 doi: 10.1029/2002JC001373. 
Godlewski, E. \& Raviart, P. -A. 1996 Numerical Approximation of Hyperbolic Systems of Conservation Laws. (Applied Mathematical Sciences), vol. 118. Springer.

HANeY, R. L. 1971 Surface thermal boundary conditions for ocean circulation models. J. Phys. Oceanogr. 1, 241-248.

Hibler, W. D. 1979 A dynamic thermodynamic sea ice model. J. Phys. Oceanogr. 9, 815-846.

Holland, P. R. \& Feltham, D. L. 2005 Frazil dynamics and precipitation in a water column with depth-dependent supercooling. J. Fluid Mech. 530, 101-124.

Hopkins, M. A. 1998 Four stages of pressure ridging. J. Geophys. Res. 103, 21883-21891.

Hunke, E. C. \& Dukowicz, J. K. 1997 An elastic viscous plastic model for sea ice dynamics. J. Phys. Oceanogr. 27, 1849-1867.

Karnovsky, N., Ainley, D. G. \& LeE, P. 2007 The impact and importance of production in polynyas to top-trophic predators: three case histories. In Polynyas: Windows to the World (ed. W. O. Smith Jr \& D. G. Barber), Elsevier Oceanography Series, vol. 74, pp. 391-410. Elsevier.

Keller, J. B. 1998 Gravity waves on ice-covered water. J. Geophys. Res. 103, 7663-7669.

Krumpen, T., Willmes, S., Morales Maqueda, M. A., HaAs, C., Hölemann, J. A., Gerdes, R. \& SCHRÖDER, D. 2011 Evaluation of a polynya flux model by means of thermal infrared satellite estimates. Ann. Glaciol. 52, 52-60.

LebedeV, V. L. 1968 Maximum size of a wind-generated lead during sea freezing. Oceanology 8, 313-318; (Engl. Transl.).

Manninen, M., Taivassalo, V. \& Kallio, S. 1996 On the mixture model for multiphase flow. Tech. Rep. VTT Publications 288. Technical Research Centre of Finland.

Marchenko, A. 2008 Thermodynamic consolidation and melting of sea ice ridges. Cold Reg. Sci. Technol. 52, 278-301.

Martin, S. 2001 Polynyas. In Encyclopedia of Ocean Sciences (ed. J. H. Steele, K. K. Turekian \& S. A. Thorpe), pp. 2241-2247. Academic Press.

Martin, S. \& Kauffman, P. 1981 A field and laboratory study of wave damping by grease ice. J. Glaciol. 27, 283-313.

Massey, B. S. 1983 Mechanics of Fluids. Van Nostrand Reinhold (International).

Miller, L. A. \& Ditullio, G. R. 2007 Gas fluxes and dynamics in polynyas. In Polynyas: Windows to the World (ed. W. O. Smith Jr \& D. G. Barber), Elsevier Oceanography Series, vol. 74, pp. 163-192. Elsevier.

MinnetT, P. J. \& KEY, E. L. 2007 Meteorology and atmosphere-surface coupling in and around polynyas. In Polynyas: Windows to the World (ed. W. O. Smith Jr \& D. G. Barber), Elsevier Oceanography Series, vol. 74, pp. 127-162. Elsevier.

Morales Maqueda, M. A. \& Holloway, G. 2006 Second-order moment advection scheme applied to arctic ocean simulation. Ocean Model. 14 (3-4), 197-221.

Morales Maqueda, M. A., Willmott, A. J., Bamber, J. L. \& Darby, M. S. 1998 An investigation of the small ice cap instability in the southern hemisphere with a coupled atmosphere-sea ice-ocean-terrestrial ice model. Clim. Dyn. 14, 329-352.

Morales Maqueda, M. A., Willmott, A. J. \& Biggs, N. R. T. 2004 Polynya dynamics: a review of observations and modelling. Rev. Geophys. 42, RG1004.

Morse, B. \& Richard, M. 2009 A field study of suspended frazil ice particles. Cold Reg. Sci. Technol. 55, 86-102.

Newyear, K. \& MARTin, S. 1997 A comparison of theory and laboratory measurements of wave propagation and attenuation in grease ice. J. Geophys. Res. 102, 25091-25099.

OU, H. W. 1988 A time-dependent model of a coastal polynya. J. Phys. Oceanogr. 18, 584-590.

Overland, J. E. \& Pease, C. H. 1988 Modelling ice dynamics of coastal seas. J. Geophys. Res. 93, 15619-15637.

Pease, C. H. 1987 The size of wind-driven coastal polynyas. J. Geophys. Res. 192, 7049-7059.

Rothrock, D. A. 1975 The enerrgetics of the plastic deformation of pack ice by ridging. J. Geophys. Res. 80, 4514-4519.

Smedrud, L. H. 2011 Grease-ice thickness parameterization. Ann. Glaciol. 52, 77-82. 
Smedrud, L. H. \& Skogseth, R. 2006 Field measurements of arctic grease ice properties and processes. Cold Reg. Sci. Technol. 44, 171-183.

STIRLING, I. 1997 The importance of polynyas, ice edges, and leads to marine mammals and birds. J. Mar. Syst. 10, 9-21.

Stoker, J. J. 1957 Water Waves. The Mathematical Theory with Applications. (Pure and Applied Mathematics), vol. IV. Interscience Publishers.

SVEnsson, U. \& OMSTEDT, A. 1998 Numerical simulations of frazil ice dynamics in the upper layers of the ocean. Cold Reg. Sci. Technol. 28, 29-44.

Tremblay, J. -E. \& Smith, W. O. 2007 Primary production and nutrient dynamics in polynyas. In Polynyas: Windows to the World (ed. W. O. Smith Jr \& D. G. Barber), Elsevier Oceanography Series, vol. 74, pp. 239-270. Elsevier.

Wilchinsky, A. V. \& Feltham, D. L. 2008 Generation of a coastal current by an antarctic polynya. J. Phys. Oceanogr. 38, 1015-1036.

Wilchinsky, A. V., Feltham, D. L. \& Miller, P. A. 2006 A multithickness sea ice model accounting for sliding friction. J. Phys. Oceanogr. 36, 1719-1738.

Williams, W. J., CARMACK, E. C. \& Ingram, R. G. 2007 Physical oceanography of polynyas. In Polynyas: Windows to the World (ed. W. O. Smith Jr \& D. G. Barber), Elsevier Oceanography Series, vol. 74, pp. 55-86. Elsevier.

Willmott, A. J., Holland, D. M. \& Morales Maqueda, M. A. 2007 Polynya modelling. In Polynyas: Windows to the World (ed. W. O. Smith Jr \& D. G. Barber), Elsevier Oceanography Series, vol. 74, pp. 87-126. Elsevier.

Winsor, P. \& BJÖRK, G. 2000 Polynya activity in the Arctic Ocean from 1958 to 1997. J. Geophys. Res. 105, 8789-8803. 